\title{
Scalable Column Concept Determination for Web Tables Using Large Knowledge Bases
}

\author{
Dong Deng ${ }^{\dagger} \quad \mathrm{Yu}$ Jiang ${ }^{\dagger} \quad$ Guoliang $\mathrm{Li}^{\dagger} \quad$ Jian $\mathrm{Li}^{\ddagger} \quad$ Cong $\mathrm{Yu}^{\#}$ \\ ${ }^{\dagger}$ Department of Computer Science, Tsinghua University, Beijing, China. \\ ${ }^{\ddagger}$ Institute for Interdisciplinary Information Sciences,Tsinghua University, Beijing, China. \\ \# Google Research, New York, USA \\ dd11@mails.thu.edu.cn, sunlight07@126.com, \{liguoliang,lijian83\}@tsinghua.edu.cn, congyu@google.com
}

\begin{abstract}
Tabular data on the Web has become a rich source of structured data that is useful for ordinary users to explore. Due to its potential, tables on the Web have recently attracted a number of studies with the goals of understanding the semantics of those Web tables and providing effective search and exploration mechanisms over them. An important part of table understanding and search is column concept determination, i.e., identifying the most appropriate concepts associated with the columns of the tables. The problem becomes especially challenging with the availability of increasingly rich knowledge bases that contain hundreds of millions of entities.

In this paper, we focus on an important instantiation of the column concept determination problem, namely, the concepts of a column are determined by fuzzy matching its cell values to the entities within a large knowledge base. We provide an efficient and scalable MapReduce-based solution that is scalable to both the number of tables and the size of the knowledge base and propose two novel techniques: knowledge concept aggregation and knowledge entity partition. We prove that both the problem of finding the optimal aggregation strategy and that of finding the optimal partition strategy are NP-hard, and propose efficient heuristic techniques by leveraging the hierarchy of the knowledge base. Experimental results on real-world datasets show that our method achieves high annotation quality and performance, and scales well.
\end{abstract}

\section{INTRODUCTION}

The Web contains a vast amount of structured data in the form of tables. According to a recent Google study [6, there are a total of 14 billion raw HTML tables and, among those, 150 million relational data tables, easily making them one of the richest structured data sources. As a result, tables on the Web have received significant interests from both industry 28, 37] and academia [19], with the focus on understanding the semantics of the tables. More recently, those tabu-

Permission to make digital or hard copies of all or part of this work for personal or classroom use is granted without fee provided that copies are not made or distributed for profit or commercial advantage and that copies bear this notice and the full citation on the first page. To copy otherwise, to republish, to post on servers or to redistribute to lists, requires prior specific permission and/or a fee. Articles from this volume were invited to present their results at The 39th International Conference on Very Large Data Bases, August 26th - 30th 2013, Riva del Garda, Trento, Italy.

Proceedings of the VLDB Endowment, Vol. 6, No. 13

Copyright 2013 VLDB Endowment 2150-8097/13/13... 10.00. lar data have begun to enrich users' search experience 13 38. For example, Google's Table Search ${ }^{*}$ are helping users browsing and finding structured data through queries such as "asian country gdp."

To leverage Web tables for applications such as table search and data integration, a number of tasks must be performed on the tables to recover their semantics. Those tasks include, but are not limited to, annotating cell values (e.g., matching the cell's textual content to an entity in a knowledge base), discovering table subjects, and identifying table schema. None of those tasks are easy because of the heterogeneous nature of data on the Web. Underneath all those tasks, one of the core table understanding tasks is column concept determination, i.e., given a column of cells within a Web table, determining the most likely concept for the column, where a concept is a type (or class) within a given knowledge base. For example, a better understanding of the dominant concept(s) of a column can help constrain cell-level annotations for cells within the column, leading to better cell annotations.

Not surprisingly, due to its importance, column concept determination is studied by both [19] and 28. In [19, column concepts are discovered as part of a graphical model that is employed to collectively learn three things at the same time: column concepts, binary relationships between multiple columns, and class labels on cells. Intuitively, this approach achieves better quality, but is in general not very scalable due to the expensive learning model being employed. In 28, column concepts are determined using a simpler majority-rule mechanism of selecting the concept(s) that the most cells in the column have been mapped to, but with a much larger knowledge base that is derived from the Web. There are several drawbacks of this approach. First, the majority-rule mechanism can lead to mistakes because of the presence of stop concepts, i.e., concepts such as "music" that can often be mapped to many textual contents indiscriminately. Second, only flat knowledge bases are considered, leaving the semantics of knowledge hierarchies underutilized. Third, only exact matching is used to map cell values to the knowledge base, and extending exact matching to approximate matching is a non-trivial task that is made more challenging by the ever increasing size of the available knowledge bases 1, 10, 26, 34.

Similar to 28, we focus on the same important instantiation of the column concept determination problem, i.e., identifying a column's top $k$ concepts based on matching its content to a large knowledge base. However, instead

\footnotetext{
http://research.google.com/tables
} 
of restricting the matching to be exact and the ranking of the concept to be majority-rule (which are what made the previous approach scalable), we aim to provide a solution that allows both fuzzy matching and ranking of the concepts based on more general similarity functions, without sacrificing scalability. This solution enables us to produce column concepts that are as good as those produced by [19], but in a much more scalable fashion.

More specifically, we identify top $k$ similar concepts from the knowledge base, where the similarity between a concept and a column is quantified based on the matching between the entities in the concept and the cell values of the column. Fuzzy matching is enabled between the cell values and knowledge entities, such that "George Bush" and "George W. Bush" can be matched. A more general set of similarity functions can be adopted to allow more flexible ranking of the candidate concepts than simple majority-rule. Both features are accomplished without compromising the scalability of the whole process.

Contributions: (1) We extend MapReduce-based similarityjoins to support scalable column concept determination. (2) We propose a knowledge concept aggregation based method which aggregates knowledge concepts into groups. Computations can then be shared for concepts in the same group. (3) We devise a knowledge entity partition based method which partitions entities into different partitions. For each column, only the necessary partitions are used to annotate the column while large numbers of unnecessary partitions are pruned. (4) We prove that both the problem of finding the optimal aggregation strategy and that of finding the optimal partition strategy are NP-hard. We utilize the knowledge hierarchy to heuristically generate high-quality groups and partitions. (5) Experimental results on realworld datasets show that our method achieves high annotation quantity and performance, and scales well.

Paper Structure: We formalize the problem in Section 2 and review related works in Section 3 A similarity-join based framework is discussed in Section 4. We propose an aggregation based method in Section 5 and a partition based method in Section 6. Experimental results are reported in Section 7. We conclude the paper in Section 8 .

\section{PRELIMINARIES}

\subsection{Problem Statement}

Data Model: A knowledge base consists of many types (or concepts). In the paper we use the terms "concepts" and "types" interchangeably. Each type has a set of large numbers of entities (or instances). The knowledge base has a hierarchy and can be modelled as a Directed Acyclic Graph. Each node in the graph is a type or an entity. Each edge between two type nodes indicates the subtype relationship of the two types. Each edge between a type node and an entity node denotes the entity belongs to the type. For example, Figure 1 shows a partial knowledge base, where the leaf-level nodes are entities, e.g., "Forrest Gump", and intermediatelevel nodes are types, e.g., "/film" and "/film/drama". Type "/film/drama" is a subtype of "/film". "Forrest Gump" and "Catch Me If You Can" are entities of type "/film/drama".

We consider a table as consisting of a sequence of columns, each of which has a set of cell values. For example, Table 1 illustrates a Web table with 4 columns.
Problem Formulation: Given a large set (tens or hundreds of millions) of tables from the Web, and a comprehensive knowledge base (with tens or hundreds of millions of entities), for each column of all the tables, efficiently identify top- $k$ types from the knowledge base that can be used to describe the column based on a user provided similarity function. More specifically, a column C can be described by a type $\mathrm{T}$ if $\mathrm{T}$ shares significant similarity with $\mathrm{C}$. Our goal is to identify top- $k$ types with the highest similarities to label all the columns, with an emphasis on efficiency and scalability. In the paper we focus on using the cell content and extracting concepts from the content of the cells. For example, the second column of the table in Table 1 should be annotated with "/film", while the third column should be annotated with "/people/director".

To better describe a column, the identified types and the column should have large similarity. Based on this observation, we utilize similarity functions to quantify the similarity between a type and a column.

\subsection{Similarity Functions}

Given a column $\mathrm{C}$ and a type $\mathrm{T}$, we define their similarity as the set similarity of C's cell-value set, denoted by $\mathrm{V}_{\mathrm{C}}$, and T's entity set, denoted by $E_{T}$. Existing set similarity can be broadly classified into two categories: exact matching similarity and fuzzy matching similarity.

Exact-matching Similarity Functions: They define the similarity based on the overlap of the two sets. We take the following well-known set similarity functions as examples.

$$
\begin{aligned}
& \text { Overlap Similarity: } \operatorname{Sim}(\mathrm{C}, \mathrm{T})=\left|\mathrm{V}_{\mathrm{C}} \cap \mathrm{E}_{\mathrm{T}}\right| \\
& \text { Jaccard Similarity: } \operatorname{Sim}(\mathrm{C}, \mathrm{T})=\frac{\left|\mathrm{V}_{\mathrm{C}} \cap \mathrm{E}_{\mathrm{T}}\right|}{\left|\mathrm{V}_{\mathrm{C}}\right|+\left|\mathrm{E}_{\mathrm{T}}\right|-\left|\mathrm{V}_{\mathrm{C}} \cap \mathrm{E}_{\mathrm{T}}\right|} . \\
& \text { Cosine Similarity: } \operatorname{Sim}(\mathrm{C}, \mathrm{T})=\frac{\left|\mathrm{V}_{\mathrm{C}} \cap \mathrm{E}_{\mathrm{T}}\right|}{\sqrt{\left|\mathrm{V}_{\mathrm{C}}\right|\left|\mathrm{E}_{\mathrm{T}}\right|}} . \\
& \text { Dice Similarity: } \operatorname{Sim}(\mathrm{C}, \mathrm{T})=\frac{2\left|\mathrm{~V}_{\mathrm{C}} \cap \mathrm{E}_{\mathrm{T}}\right|}{\left|\mathrm{V}_{\mathrm{C}}\right|+\left|\mathrm{E}_{\mathrm{T}}\right|} . \\
& \text { Weighted Jaccard Similarity: } \operatorname{Sim}(\mathrm{C}, \mathrm{T})=\frac{\sum_{t \in \mathrm{V}_{\mathrm{C}} \cap \mathrm{E}_{\mathrm{T}}} w_{t}}{\sum_{t \in \mathrm{V}_{\mathrm{C}} \cup \mathrm{E}_{\mathrm{T}}} w_{t}}, \\
& \text { where } w_{t} \text { is the weight of a cell value or an entity. }
\end{aligned}
$$

Fuzzy-matching Similarity Functions: Exact matching similarity functions can be restricted as they cannot capture fuzzy matching between cell values and entities. However in many real-world applications, we need to accommodate fuzzy matching 7]. For example, many Web tables contain typing errors and an entity has different representations. Furthermore, some columns may match multiple types, e.g., movie and director. Thus we need to enable fuzzy matching between an entity and a cell value.

Before we introduce fuzzy-matching similarity functions, we first define some notations. Let $\operatorname{ES} \operatorname{Sim}(v, e)$ denote the similarity between a cell value $v$ and an entity $e$. We can use existing similarity functions to quantify their similarity, e.g., Jaccard, Cosine, and Edit Distance Given a type $\mathrm{T}$ and a column $\mathrm{C}$, a set of $\langle$ cell value, entity $\rangle$ pairs, denoted by $\mathcal{M}=\left\{\left(v_{i}, e_{j}\right) \mid v_{i} \in \mathrm{V}_{\mathrm{C}}, e_{j} \in \mathrm{E}_{\mathrm{T}}\right\}$, is a matching if each $v_{i}$ and $e_{j}$ appears at most once in $\mathcal{M}$. The maximum matching $\mathcal{M}_{\max }$ is the matching with the maximum value $\sum_{\left(v_{i}, e_{j}\right) \in \mathcal{M}_{\text {max }}} \operatorname{ESIM}\left(v_{i}, e_{j}\right)$. We can use existing algorithms 31 to compute the maximum matching. We use

\footnotetext{
$\dagger^{\top}$ The edit distance between two elements is the minimum number of edit operations (insertion, deletion, substitution) needed to transform one element to another.
} 
Table 1: Example table from the Web (http://celebrity.psyphil.com/actor/Tom-Hanks/filmography).

\begin{tabular}{|l|l|l|l|}
\hline 1994 & Forrest Gump & Robert Zemeckis & Tom Hanks, Robin Wright, Sally Field, ... \\
\hline 1998 & Saving Private Ryan & Steven Spielberg & Tom Hanks, Tom Sizemore, Matt Damon, ... \\
\hline 1999 & The Green Mile & Frank Darabont & Tom Hanks, David Morse, Bonnie Hunt, ... \\
\hline 1995 & Toy Story & John Lasseter & Tom Hanks, Tim Allen, Wallace Shawn, ... \\
\hline 2002 & Catch Me If You Can & Steven Spielberg & Tom Hanks, Christopher Walken, Leonardo DiCaprio, ... \\
\hline 2006 & The Da Vinci Code & Ron Howard & Tom Hanks, Ian McKellen, Jean Reno, .... \\
\hline
\end{tabular}

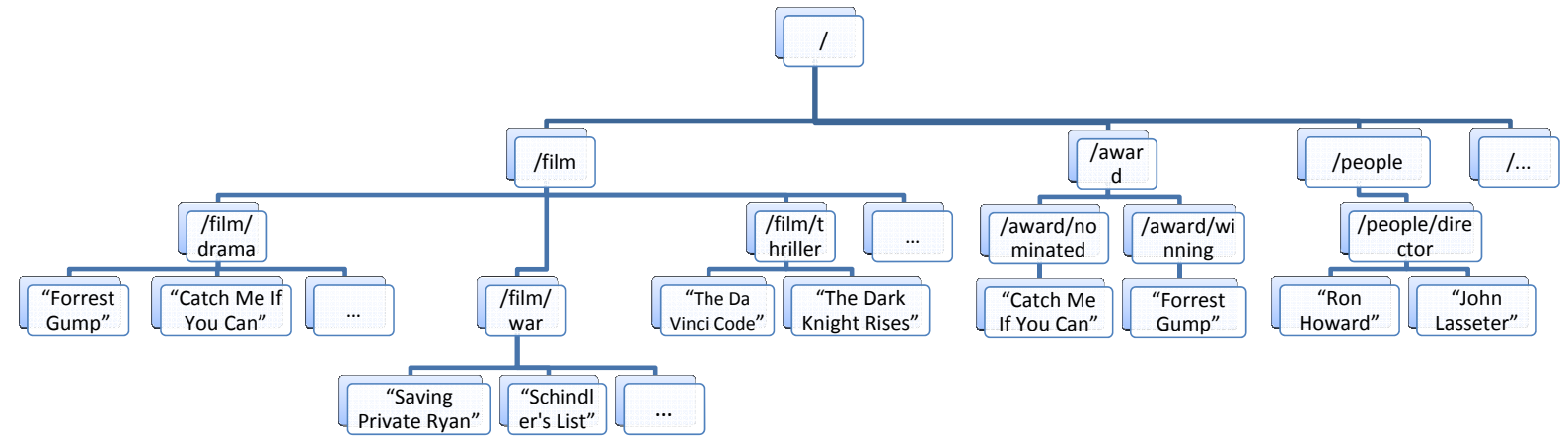

Figure 1: Example knowledge base based on Wikipedia.

$\mathrm{V}_{\mathrm{C}} \widetilde{\cap} \mathrm{E}_{\mathrm{T}}$ to denote the maximum matching, called fuzzy overlap of $\mathrm{T}$ and $\mathrm{C}$, and $\left|\mathrm{V}_{\mathrm{C}} \widetilde{\cap} \mathrm{E}_{\mathrm{T}}\right|$ to denote its value.

We can extend exact-matching functions by replacing $\mathrm{V}_{\mathrm{C}} \cap E_{\mathrm{T}}$ with $\mathrm{V}_{\mathrm{C}} \widetilde{\cap} E_{\mathrm{T}}$ to define fuzzy-matching functions. Next we take fuzzy Jaccard similarity as an example.

$$
\text { Fuzzy Jaccard Similarity: } \operatorname{Sim}(\mathrm{C}, \mathrm{T})=\frac{\left|\mathrm{V}_{\mathrm{C}} \widetilde{\cap}_{\mathrm{T}}\right|}{\left|\mathrm{V}_{\mathrm{C}}\right|+\left|\mathrm{E}_{\mathrm{T}}\right|-\left|\mathrm{V}_{\mathrm{C}} \widetilde{\cap} \mathrm{E}_{\mathrm{T}}\right|} .
$$

For example, consider a column with two cell values $\{$ "Shark Night 3D", "Da Vinci Code" $\}$ and a type with two entities \{ "Shark Night", "The Da Vinci Code" $\}$. Suppose we use the Jaccard similarity. "Shark Night 3D" and "Shark Night" are fuzzy matching with similarity 0.67. "Da Vinci Code" and "The Da Vinci Code" are fuzzy matching with similarity 0.75. Thus the fuzzy overlap size is $0.67+0.75=1.42$ and the fuzzy Jaccard similarity is $\frac{1.42}{2+2-1.42}=0.55$.

Scalability Requirement: Since there are hundreds of millions of columns and millions of entities, existing machine learning based methods 19, 28, are neither efficient nor scalable for such large datasets and it calls for scalable methods. To this end, we extend existing MapReduce-based similarity-join methods and develop effective techniques to support efficient and scalable column concept determination, while maintaining the annotation quality.

\section{RELATED WORKS}

MapReduce: We adopt the MapReduce framework to address the scalability challenge. MapReduce 8 is a dominant framework to support data-intensive parallel computation in shared-nothing clusters. In MapReduce, data is represented as $\langle$ key, value $\rangle$ pairs and stored in a distributed file system (DFS) across different cluster nodes. MapReduce contains two core functions: Map and Reduce. The map phase loads and processes different partitions of the input data in parallel and outputs 〈key, value〉 pairs. The pairs are then hashpartitioned, sorted by the key, and sent across the cluster in a shuffle phase. The pairs that share the same key are merged in a sorted order and sent to the same reduce node, which then processes the pairs and writes new 〈key, value〉 pairs to files on the DFS.

Knowledge Bases: DBPedia 3] and Freebase 5] are similar projects that extract information from Wikipedia to create an extensive structured knowledge base and supplement those data with knowledge that are collaboratively created by thousands of data-loving users. Freebase contained about 2 thousand types and 40 million entities. Probase 34 is a universal, probabilistic taxonomy which is harnessed from billions of Web pages using "isA" and "such as" patterns. Probase includes more than 2.7 million types and 22 million entities. Finally, Yago 26 is a large semantic knowledge base which is derived from Wikipedia and WordNet, and it contains 0.3 million types and 9 million entities.

Annotating Web Tables: Google's WebTables project 6 is the first to start exploring table data on the Web, which has been followed up by a number of other studies $9,19,28$ 37. Google has since launched a Table Search project 38 at research.google.com/tables, where external users can explore the table data. Google has also started exploring stitching similar tables on the same page 20 to provide better utility from the table data. At the core of managing Web table data is the annotation problem, i.e., finding meanings for the cells and columns in the table. Existing studies used machine learning techniques to annotate tables 19 that are neither as scalable nor as efficient as the approaches we present in this paper. Other relevant studies (although those do not address the column concept identification problem) include: Yakout et. al. 37 studied how to augment entities with attribute values and discovering attributes using Web tables. Pimplikar et. al. 22 answered table queries based on column keywords. Quercini et. al. 23 utilized search engines to discover and annotate entities in Web tables. Hignette 15 annotated tables with ontology concepts based on relevance degrees. Wang et. al. 33 utilized interconnections of Probase to understand Web tables.

Extracting Table Data into RDF Data: There are many studies on extracting table data into RDF data 12 14. Guo et. al. 12 used a schema mapping technique to extract and integrate data from tables into RDF. Han et. al. 14 studied how to translate spreadsheet data into RDF. Assem et. al. 27. studied how to annotate and covert quantitative data tables into RDF. Different from these methods, our work focuses on making fuzzy matching based annotation techniques more scalable and efficient. How to apply our technique to the annotation techniques proposed by those studies is a challenge that we intend to pursue in the future. 
Similarity Join: There are many recent studies on similarity join, which, given two sets of objects, find all similar object pairs based on a given similarity function and a threshold [35, 24, 36, 11, 2, 29, 18, 30, 32. Vernica et al. 29. and Metwally et al. 21 proposed a MapReduce framework to support similarity joins. However they required a similarity threshold and their techniques cannot be extended to support our ranking problem. Kim et al. 17] studied parallel algorithm for top- $k$ similarity join with Euclidean distance constraint using MapReduce while we focus on finding top- $k$ types for each column. These methods do not allow fuzzy matching between entities and cell values.

\section{SIMILARITY-JOIN FRAMEWORK}

In this section, we extend MapReduce-based similarity joins to support scalable column concept determination. We first take the overlap similarity as an example in Section 4.1 and then extend it to support other functions in Section 4.2 .

\subsection{Framework for Overlap Similarity}

If we use a type to annotate a column, the type should share at least one common entity/cell value with the column. Thus for each column, we first find the types that share common entities with the column and then rank these types to return top- $k$ types. To implement this idea in MapReduce, we introduce a two-stage similarity-join based framework. In the first stage, for each column, we identify the types which share at least one common entity/cell value with the column. In the second stage, we count the number of their shared entities/cell values and retrieve top $k$ types with the largest similarity. Algorithm 1 illustrates the pseudo-code.

Stage 1: For each column, find the list of types that share at least one entity/cell value with the column.

Map: $\left\langle\mathrm{C},\left\{v_{1}, v_{2}, \ldots\right\}\right\rangle \rightarrow\left\langle v_{i}, \mathrm{C}\right\rangle .\left\langle\mathrm{T},\left\{e_{1}, e_{2}, \ldots\right\}\right\rangle \rightarrow\left\langle e_{i}, \mathrm{~T}\right\rangle$.

It takes as input a sequence of columns (each column includes a set of cell values) from Web tables and a set of types (each type includes a set of entities) from a knowledge base. For each column $\mathrm{C}$, it outputs key-value pairs $\left\langle v_{i}, \mathrm{C}\right\rangle$ where $v_{i}$ is any cell value of $\mathrm{C}$. For each type $\mathrm{T}$, it outputs key-value pairs $\left\langle e_{i}, \mathrm{~T}\right\rangle$ where $e_{i}$ is any entity of $\mathrm{T}$.

Reduce: $\left\langle v_{i}=e_{i}, \operatorname{list}(\mathrm{C} / \mathrm{T})\right\rangle \rightarrow\langle\mathrm{C}, \operatorname{list}(\mathrm{T})\rangle$.

It takes as input a set of key-value pairs $\left\langle v_{i}=e_{i}, \operatorname{list}(\mathrm{C} / \mathrm{T})\right\rangle$, where $v_{i}=e_{i}$ is a cell value/an entity, and $\operatorname{list}(\mathrm{C} / \mathrm{T})$ is a list of columns/types that contain the cell value/entity. The Reducer separates list(C/T) into a list of columns, denoted by $\operatorname{list}(\mathrm{C})$ and a list of types, denoted by $\operatorname{list}(\mathrm{T})$. (We use a flag to differentiate $\mathrm{C} / \mathrm{T}$ to be a column or a type.) For each column $\mathrm{C}$ in $\operatorname{list}(\mathrm{C})$, it outputs a key-value pair $\langle\mathrm{C}, \operatorname{list}(\mathrm{T})\rangle$.

Combine: We can use Combine to do a local reduce. As it is not a focus of this paper, we will not discuss the details.

Stage 2: Compute top- $k$ types of every column.

Map: $\langle\mathrm{C}, \operatorname{list}(\mathrm{T})\rangle \rightarrow\langle\mathrm{C}, \operatorname{list}(\mathrm{T})\rangle$.

It takes as input key-value pairs $\langle\mathrm{C}, \operatorname{list}(\mathrm{T})\rangle$, where $\operatorname{list}(\mathrm{T})$ is a list of types that share an entity/cell value with C. It outputs the same key-value pairs as input.

Reduce: $\langle\mathrm{C}, \operatorname{list}(\operatorname{list}(\mathrm{T}))\rangle \rightarrow\langle\mathrm{C}, \operatorname{topk}(\mathrm{T})\rangle$.

It takes as input key-value pairs $\langle\mathrm{C}, \operatorname{list}(\operatorname{list}(\mathrm{T}))\rangle$, where list $($ list $(\mathrm{T}))$ is a list of lists of types that share common entities/cell values with $\mathrm{C}$. We use a hash based method to compute the overlap similarity of $\mathrm{C}$ and a type $\mathrm{T}$ in $\operatorname{list}(\operatorname{list}(\mathrm{T}))$. We first initialize a hash table. Then for each type in $\operatorname{list}(\operatorname{list}(\mathrm{T})$

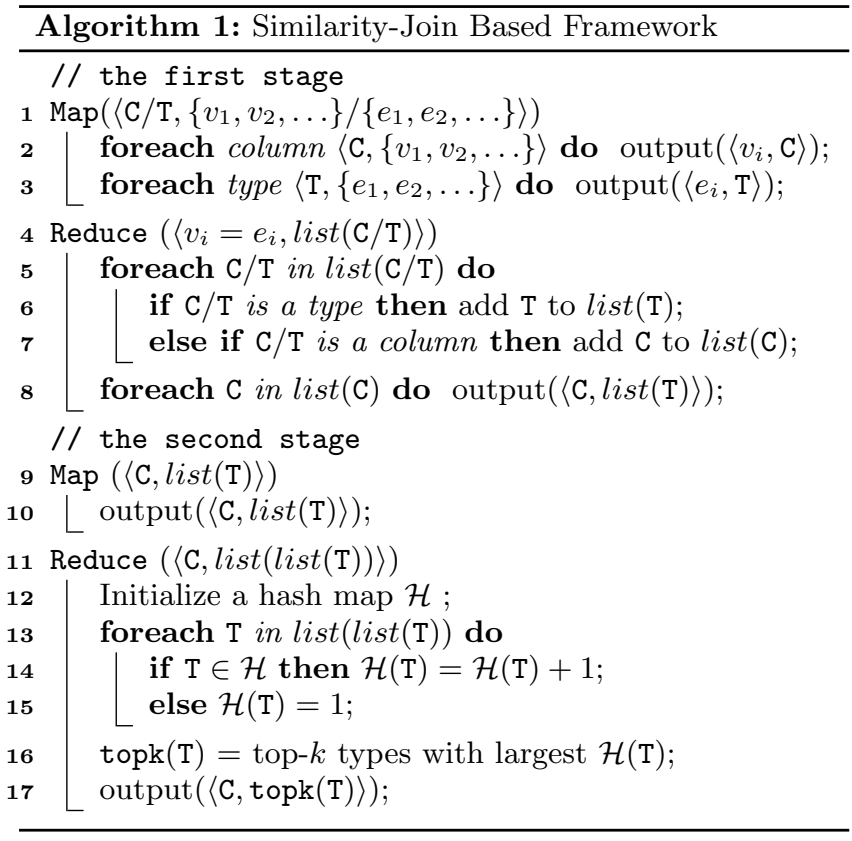

if it is not in the hash table, we add it into the hash table and set the occurrence number as 1 ; otherwise we increase the number by 1 . After scanning all types, the occurrence number of a type is exactly the overlap similarity of the type with the column. Based on the number, we rank the types and add the top- $k$ types with the largest overlap similarity into topk(T). Finally it outputs key-value pairs $\langle\mathrm{C}$, topk $(\mathrm{T})\rangle$.

EXAMPLE 1. Figure 2 shows four columns and four types. Figure 3 illustrates a running example that uses our framework to label each column. In the first stage, the Map step reads the column file and the type file. For each column (type), it outputs 〈cell value, column $\rangle(\langle$ entity, type $\rangle)$ pairs. For $\mathrm{C}_{1}$, it outputs six key-value pairs $\left\langle v_{1}, \mathrm{C}_{1}\right\rangle, \ldots,\left\langle v_{6}, \mathrm{C}_{1}\right\rangle$. For $\mathrm{T}_{2}$, it outputs two key-value pairs $\left\langle e_{1}, \mathrm{~T}_{2}\right\rangle,\left\langle e_{2}, \mathrm{~T}_{2}\right\rangle$. In the Reduce step, it reads the key-value pairs grouped by the key(cell value or entity). Consider $\left\langle v_{1}=e_{1},\left\{\mathrm{C}_{1}, \mathrm{C}_{2}, \mathrm{~T}_{1}, \mathrm{~T}_{2}\right\}\right\rangle$. $\mathrm{T}_{1}, \mathrm{~T}_{2}$ share an entity $e_{1}$ with $\mathrm{C}_{1}, \mathrm{C}_{2}$. It outputs $\left\langle\mathrm{C}_{1},\left\{\mathrm{~T}_{1}, \mathrm{~T}_{2}\right\}\right\rangle$ and $\left\langle\mathrm{C}_{2},\left\{\mathrm{~T}_{1}, \mathrm{~T}_{2}\right\}\right\rangle$. In the second stage, the Map step outputs the same key-value pairs as the input. The Reduce step computes top- $k$ types for each column by counting the occurrence number of each type in the key-value pair. Consider $\left\langle\mathrm{C}_{1},\left\{\left\{\mathrm{~T}_{1}, \mathrm{~T}_{2}\right\},\left\{\mathrm{T}_{1}, \mathrm{~T}_{2}\right\},\left\{\mathrm{T}_{1}, \mathrm{~T}_{3}\right\},\left\{\mathrm{T}_{1}, \mathrm{~T}_{3}\right\}\right\}\right\rangle . \mathrm{T}_{1}$ appears four times, and $\mathrm{T}_{2}$ and $\mathrm{T}_{3}$ appear twice. Thus the top-1 type for $\mathrm{C}_{1}$ is $\mathrm{T}_{1}$. Similarity the top-1 type for $C_{2}\left(\mathrm{C}_{3}\right)$ is $\mathrm{T}_{2}\left(\mathrm{~T}_{3}\right) . W e$ cannot label $\mathrm{C}_{4}$ as it does not share any entity with any type.

\subsection{Extension to Other Functions}

Exact-matching Similarity Functions: Different from the overlap similarity, besides $\left|\mathrm{V}_{\mathrm{C}} \cap \mathrm{E}_{\mathrm{T}}\right|$, other exact-matching functions, e.g., weighted Jaccard, also rely on $\left|\mathrm{V}_{\mathrm{C}}\right|,\left|\mathrm{E}_{\mathrm{T}}\right|$, and the weight of each entity/cell value. To support these functions, we add these parameters into the key-value pairs, and in the Reduce step of the second stage, we compute the similarity based on these parameters. Thus we can extend this framework to support other exact-matching functions.

Fuzzy-matching Similarity Functions: To support fuzzymatching functions, we slightly modify the framework. For 


\begin{tabular}{|l|l|}
\hline \multicolumn{2}{|c|}{ Column C 1} \\
\hline$v_{1}$ & Forrest Gump \\
\hline$v_{2}$ & Catch Me If You Can \\
\hline$v_{3}$ & Saving Private Ryan \\
\hline$v_{4}$ & Schindler's List \\
\hline$v_{5}$ & Shark Night 3D \\
\hline$v_{6}$ & Da Vinci Code \\
\hline$v_{7}$ & The Terminal \\
\hline$v_{8}$ & Full Metal Jacket \\
\hline \hline \multicolumn{2}{|c|}{ Column C 2} \\
\hline$v_{1}$ & Forrest Gump \\
\hline$v_{2}$ & Catch Me If You Can \\
\hline$v_{7}$ & The Terminal \\
\hline \hline \multicolumn{2}{|c|}{ Column C 3} \\
\hline$v_{3}$ & Saving Private Ryan \\
\hline$v_{4}$ & Schindler's List \\
\hline$v_{8}$ & Full Metal Jacket \\
\hline \hline \multicolumn{2}{|c|}{ Column C 4} \\
\hline$v_{5}$ & Shark Night 3D \\
\hline$v_{6}$ & Da Vinci Code \\
\hline
\end{tabular}

\begin{tabular}{|c|c|}
\hline \multicolumn{2}{|r|}{ Type $\mathrm{T}_{1}(/ \mathrm{film})$} \\
\hline$e_{1}$ & Forrest Gump \\
\hline$e_{2}$ & Catch Me If You Can \\
\hline$e_{3}$ & Saving Private Ryan \\
\hline$e_{4}$ & Schindler's List \\
\hline$e_{5}$ & Shark Night \\
\hline$e_{6}$ & The Da Vinci Code \\
\hline$e_{7}$ & The Green Mile \\
\hline$e_{8}$ & Heartbreak Ridge \\
\hline \multicolumn{2}{|c|}{ Type $\mathrm{T}_{2}$ (/film/drama) } \\
\hline$e_{1}$ & Forrest Gump \\
\hline$e_{2}$ & Catch Me If You Can \\
\hline$e_{7}$ & The Green Mile \\
\hline \multicolumn{2}{|r|}{ Type $\mathrm{T}_{3}$ (/film/war) } \\
\hline$e_{3}$ & Saving Private Ryan \\
\hline$e_{4}$ & Schindler's List \\
\hline$e_{8}$ & Heartbreak Ridge \\
\hline \multicolumn{2}{|c|}{ Type $\mathrm{T}_{4}$ (/film/thriller) } \\
\hline$e_{5}$ & Shark Night \\
\hline$e_{6}$ & The $\mathrm{Da} \mathrm{Vi}$ \\
\hline
\end{tabular}

Column
\begin{tabular}{|l|l|}
\hline$C_{1}$ & $V_{1} V_{2} V_{3} \ldots V_{8}$ \\
\hline$C_{2}$ & $V_{1} V_{2} V_{7}$ \\
\hline$C_{3}$ & $V_{3} V_{4} V_{8}$ \\
\hline$C_{4}$ & $V_{5} V_{6}$ \\
\hline
\end{tabular}
\begin{tabular}{|l|l|}
\hline & $T y p e$ \\
\hline$T_{1}$ & $e_{1} e_{2} e_{3} \ldots e_{8}$ \\
\hline$T_{2}$ & $e_{1} e_{2} e_{7}$ \\
\hline$T_{3}$ & $e_{3} e_{4} e_{8}$ \\
\hline$T_{4}$ & $e_{5} e_{6}$ \\
\hline
\end{tabular}
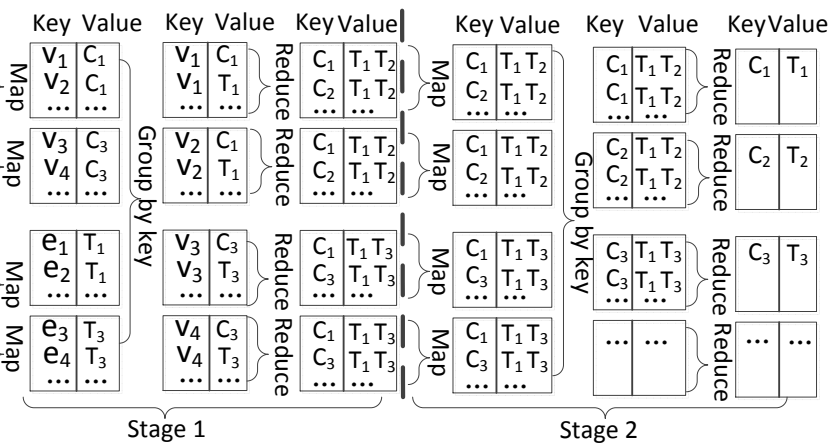

Figure 2: Example columns and types.

each cell value (or entity), we generate its signatures. A cell value and an entity is similar only if they share at least one common signature. There are many signature strategies, e.g., $q$-gram 11 and prefix signature 29]. We can use any of them in our method. Next we modify the basic framework to support fuzzy-matching functions based on the signatures. Stage 1: For each column, find the list of types that share at least one common signature with the column.

Map: $\left\langle\mathrm{C},\left\{v_{1}, \ldots\right\}\right\rangle \rightarrow\left\langle\right.$ sig, $\left.\left\langle\mathrm{C}, v_{i}\right\rangle\right\rangle .\left\langle\mathrm{T},\left\{e_{1}, \ldots\right\}\right\rangle \rightarrow\left\langle\right.$ sig, $\left.\left\langle\mathrm{T}, e_{j}\right\rangle\right\rangle$.

For each column $\mathrm{C}$, for each cell value $v_{i}$, it computes the signatures and for each signature sig, generates keyvalue pairs, $\left\langle\mathrm{sig},\left\langle\mathrm{C}, v_{i}\right\rangle\right\rangle$. For each type $\mathrm{T}$, for each entity $e_{j}$, it computes the signatures and for each signature sig, generates key-value pairs $\left\langle\right.$ sig, $\left.\left\langle\mathrm{T}, e_{j}\right\rangle\right\rangle$.

Reduce: $\left\langle\right.$ sig, $\left.\operatorname{list}\left(\left\langle\mathrm{C}, v_{i}\right\rangle /\left\langle\mathrm{T}, e_{j}\right\rangle\right)\right\rangle \rightarrow\left\langle\mathrm{C}, \operatorname{list}\left(\left\langle\mathrm{T}, v_{i}, e_{j}\right\rangle\right)\right\rangle$.

It separates the list into the column list and the type list. For each column, for each type, if the column has a cell value that shares a common signature with an entity of the type, it adds the type into a type list $\operatorname{list}\left(\left\langle\mathrm{T}, v_{i}, e_{j}\right\rangle\right)$. Finally it outputs key-value pairs $\left\langle\mathrm{C}, \operatorname{list}\left(\left\langle\mathrm{T}, v_{i}, e_{j}\right\rangle\right)\right\rangle$.

Stage 2: Compute top- $k$ types of every column.

Map: $\left\langle\mathrm{C}, \operatorname{list}\left(\left\langle\mathrm{T}, v_{i}, e_{j}\right\rangle\right)\right\rangle \rightarrow\left\langle\mathrm{C}, \operatorname{list}\left(\left\langle\mathrm{T}, v_{i}, e_{j}\right\rangle\right)\right\rangle$.

It outputs the same key-value pairs as the input.

Reduce: $\left\langle\mathrm{C}\right.$, list $\left.\left(\operatorname{list}\left(\left\langle\mathrm{T}, v_{i}, e_{j}\right\rangle\right)\right)\right\rangle \rightarrow\langle\mathrm{C}, \operatorname{topk}(\mathrm{T})\rangle$.

For each column, it gets a list of lists of types. Then it computes the fuzzy-matching similarity using existing algorithms 31. Based on the fuzzy-matching similarity, we rank the types and identify the top- $k$ types with the largest fuzzy-matching similarity to annotate the column.

ExAmple 2. Consider column $\mathrm{C}_{4}$ and type $\mathrm{T}_{4}$. Suppose the signatures of $v_{5}=$ "Shark Night 3D" and $e_{5}=$ "Shark Night" are sig $=$ "Shark". The signatures of $v_{6}=$ "Da Vinci Code" and $e_{6}=$ "The Da Vinci Code" are sig $g_{2}=\{$ "Vinci" $\}$. In the Map step of the first stage, we generate pairs $\left\langle s i g_{1},\left\langle\mathrm{C}_{4}, v_{5}\right\rangle\right\rangle$ and $\left\langle\right.$ sig $\left._{2},\left\langle\mathrm{C}_{4}, v_{6}\right\rangle\right\rangle$ for $\mathrm{C}_{4}$ and $\left\langle\right.$ sig $\left._{1},\left\langle\mathrm{~T}_{4}, v_{5}\right\rangle\right\rangle$ and $\left\langle\right.$ sig $\left._{2},\left\langle\mathrm{~T}_{4}, v_{6}\right\rangle\right\rangle$ for $\mathrm{T}_{4}$. In the Reduce step, we generate $\left\langle\mathrm{C}_{4},\left\langle\mathrm{~T}_{4}, v_{5}, e_{5}\right\rangle\right\rangle$ and $\left\langle\mathrm{C}_{4},\left\langle\mathrm{~T}_{4}, v_{6}, e_{6}\right\rangle\right\rangle$. In the Reduce step of the second stage, we compute the fuzzy similarity. The top-1 type of $\mathrm{C}_{4}$ is $\mathrm{T}_{4}$. Thus the fuzzy matching functions can annotate $\mathrm{C}_{4}$ by tolerating inconsistencies between entities and cell values.

\subsection{Observations}

Optimization Goal: To improve the performance, our optimization goal is to reduce the number of key-value pairs. Although we can use the prefix-filtering technique 29], it has the following problems. First, it depends on a given threshold and cannot support our top- $k$ problem. Second, it involves other expensive overhead 29], e.g., pre-defining a global order and selecting high-quality prefixes based on the global order. To address these problems, we propose effective pruning technique based on the following observations.

Observation 1: We can aggregate the types into different groups. For each entity, to generate the key-value pairs, we use a group to replace the types in the group. Since each group contains multiple types, this method can reduce the number of key-value pairs. In addition, a group may be used by multiple entities, and we can share the computations for the types in the same group. We will discuss how to utilize groups to label columns in Section 5 .

Observation 2: We can partition the cell values into different partitions. For each column, we utilize the partitions to generate its key-value pairs. Since each partition contains multiple cell values, this method can reduce the number of key-value pairs. Moreover, if the cell values of a column fall into one partition, we can utilize the partition to directly annotate the column and prune other unnecessary partitions. We will discuss the details in Section 6.

In the following sections, we focus on improving the efficiency and scalability. For simplicity, we take overlap similarity as an example, and our techniques can be easily applied to other similarity functions.

\section{KNOWLEDGE TYPE AGGREGATION}

For each entity, we want to reduce the number of keyvalue pairs produced for the entity. Let $\mathcal{L}(e)$ denote the list of types that contain entity $e$. We aggregate types in $\mathcal{L}(e)$ and generate several disjoint groups $\mathcal{G}_{1}(e), \mathcal{G}_{2}(e), \ldots, \mathcal{G}_{l}(e)$. Instead of producing key-value pairs $\langle e, \mathrm{~T}\rangle$ for every type $\mathrm{T} \in$ $\mathcal{L}(e)$, we generate key-value pairs $\left\langle e, \mathcal{G}_{i}(e)\right\rangle$ for every group $\mathcal{G}_{i}(e)$ for $1 \leq i \leq l$. That is we use group $\mathcal{G}_{i}(e)$ to replace (multiple) types in the group. Obviously this method can reduce the number of key-value pairs and can improve the performance. There are several research challenges in this 
type aggregation based method. The first one is how to utilize the groups to compute top- $k$ types for each column. We formally introduce an aggregation framework to address this issue in Section 5.1 The second challenge is to quantify different aggregation strategies. We propose a cost based model to analyze the group quality in Section 5.2 The third one is to efficiently generate high-quality groups. We devise efficient algorithms to address this challenge in Section 5.3 .

\subsection{Aggregation Framework}

Given a knowledge base, for each entity $e$, we aggregate the types in its inverted list $\mathcal{L}(e)$ and partition them into $l$ disjoint groups $\mathcal{G}_{1}(e), \mathcal{G}_{2}(e), \ldots, \mathcal{G}_{l}(e)$, such that

(1) Subset: $\mathcal{G}_{i}(e)$ is a subset of $\mathcal{L}(e)$ for $1 \leq i \leq l$;

(2) Completeness: $\cup_{1 \leq i \leq l} \mathcal{G}_{i}(e)=\mathcal{L}(e)$; and

(3) Disjoint: $\mathcal{G}_{i}(e) \cap \mathcal{G}_{j}(e)=\phi$ for $i \neq j$.

Let $\mathcal{G}_{\mathcal{T}}=\left\{\mathcal{G}_{1}, \mathcal{G}_{2}, \ldots, \mathcal{G}_{g}\right\}$ denote the set of distinct groups for all entities. Let $\mathcal{H}_{\mathcal{T}}^{\mathcal{G}}$ denote a hash table from the distinct groups to lists of types, i.e., each group $\mathcal{G}_{i}$ is associated with a list of types contained in $\mathcal{G}_{i}, \mathcal{H}_{\mathcal{T}}^{\mathcal{G}}\left(\mathcal{G}_{i}\right)$.

For example, consider the types and entities in Figure 2 . $\mathcal{L}\left(e_{1}\right)=\left\{\mathrm{T}_{1}, \mathrm{~T}_{2}\right\} . \quad \mathcal{L}\left(e_{2}\right)=\left\{\mathrm{T}_{1}, \mathrm{~T}_{2}\right\} . \quad \mathcal{L}\left(e_{3}\right)=\left\{\mathrm{T}_{1}, \mathrm{~T}_{3}\right\}$. $\mathcal{L}\left(e_{4}\right)=\left\{\mathrm{T}_{1}, \mathrm{~T}_{3}\right\} . \quad \mathcal{L}\left(e_{5}\right)=\left\{\mathrm{T}_{1}, \mathrm{~T}_{4}\right\} . \quad \mathcal{L}\left(e_{6}\right)=\left\{\mathrm{T}_{1}, \mathrm{~T}_{4}\right\}$. $\mathcal{L}\left(e_{7}\right)=\left\{\mathrm{T}_{1}, \mathrm{~T}_{2}\right\} . \quad \mathcal{L}\left(e_{8}\right)=\left\{\mathrm{T}_{1}, \mathrm{~T}_{3}\right\} . \quad$ The basic framework will generate 16 key-value pairs. Suppose we aggregate them into three groups, $\mathcal{G}_{1}=\left\{\mathrm{T}_{1}, \mathrm{~T}_{2}\right\}, \mathcal{G}_{2}=\left\{\mathrm{T}_{1}, \mathrm{~T}_{3}\right\}, \mathcal{G}_{3}=$ $\left\{\mathrm{T}_{1}, \mathrm{~T}_{4}\right\}$. We generate 8 key-value pairs $\left\langle e_{1}, \mathcal{G}_{1}\right\rangle,\left\langle e_{2}, \mathcal{G}_{1}\right\rangle$, $\left\langle e_{3}, \mathcal{G}_{2}\right\rangle,\left\langle e_{4}, \mathcal{G}_{2}\right\rangle,\left\langle e_{5}, \mathcal{G}_{3}\right\rangle,\left\langle e_{6}, \mathcal{G}_{3}\right\rangle,\left\langle e_{7}, \mathcal{G}_{1}\right\rangle$, and $\left\langle e_{8}, \mathcal{G}_{2}\right\rangle$.

Suppose we can get the groups of each entity, i.e., $\left\langle e,\left\{\mathcal{G}_{1}(e)\right.\right.$, $\left.\mathcal{G}_{2}(e), \ldots, \mathcal{G}_{l}(e)\right\}$. We will discuss how to generate the highquality groups in Section 5.3 Next we discuss how to use the groups to compute top- $k$ types of each column. We still employ a two-stage MapReduce framework. Algorithm 2 illustrates the pseudo-code.

Stage 1: For each column, find the list of types that share at least one entity/cell value with the column.

Map: $\left\langle\mathrm{C},\left\{v_{1}, v_{2}, \ldots\right\}\right\rangle \rightarrow\left\langle v_{i}, \mathrm{C}\right\rangle .\left\langle e,\left\{\mathcal{G}_{1}(e), \ldots, \mathcal{G}_{l}(e)\right\}\right\rangle \rightarrow\left\langle e, \mathcal{G}_{i}(e)\right\rangle$

The input and output for the columns are the same as those of the basic framework. For the types, the input is a set of key-value pairs $\left\langle e,\left\{\mathcal{G}_{1}(e), \mathcal{G}_{2}(e), \ldots, \mathcal{G}_{l}(e)\right\}\right\rangle$. For each entity, it outputs key-value pairs $\left\langle e, \mathcal{G}_{i}(e)\right\rangle$ for $1 \leq i \leq l$.

Reduce: $\langle v=e, \operatorname{list}(\mathrm{C} / \mathcal{G})\rangle \rightarrow\langle\mathrm{C}, \operatorname{list}(\mathcal{G})\rangle$.

Different from the basic framework, for each column, it outputs key-value pairs $\langle\mathrm{C}, \operatorname{list}(\mathcal{G})\rangle$, where $\mathcal{G}$ is a group which has a type sharing a common entity with $\mathrm{C}$.

Stage 2: Compute top- $k$ types of every column.

Map: $\langle\mathrm{C}, \operatorname{list}(\mathcal{G})\rangle \rightarrow\langle\mathrm{C}, \operatorname{list}(\mathcal{G})\rangle$.

Similarly it outputs key-value pairs $\langle\mathrm{C}, \operatorname{list}(\mathcal{G})\rangle$.

Reduce: $\langle\mathrm{C}, \operatorname{list}(\operatorname{list}(\mathcal{G}))\rangle \rightarrow\langle\mathrm{C}, \operatorname{topk}(\mathrm{T})\rangle$.

For each column $\mathrm{C}$, it gets a list of lists of groups. We still use a hash based method to compute the overlap similarity of $\mathrm{C}$ and a type $\mathrm{T} \in \mathcal{G} \in \operatorname{list}(\operatorname{list}(\mathcal{G}))$. We first initialize a hash table. Then for each group $\mathcal{G}$ in $\operatorname{list}(\operatorname{list}(\mathcal{G}))$, we get the list of types in the group, i.e., $\mathcal{H}_{\mathcal{T}}^{\mathcal{G}}(\mathcal{G})$. For each type in $\mathcal{H}_{\mathcal{T}}^{\mathcal{G}}(\mathcal{G})$, we compute its occurrence number similar as the basic framework. Finally we identify top- $k$ types with the largest overlap similarity and add them into topk $(\mathrm{T})$.

ReduceSetup: To get $\mathcal{H}_{\mathcal{T}}^{\mathcal{G}}$ in each reducer, we take $\mathcal{H}_{\mathcal{T}}^{\mathcal{G}}$ as the side data which is distributed to each node of the cluster.

The aggregation based method can correctly identify the top- $k$ types for each column as formalized in Theorem 1 .

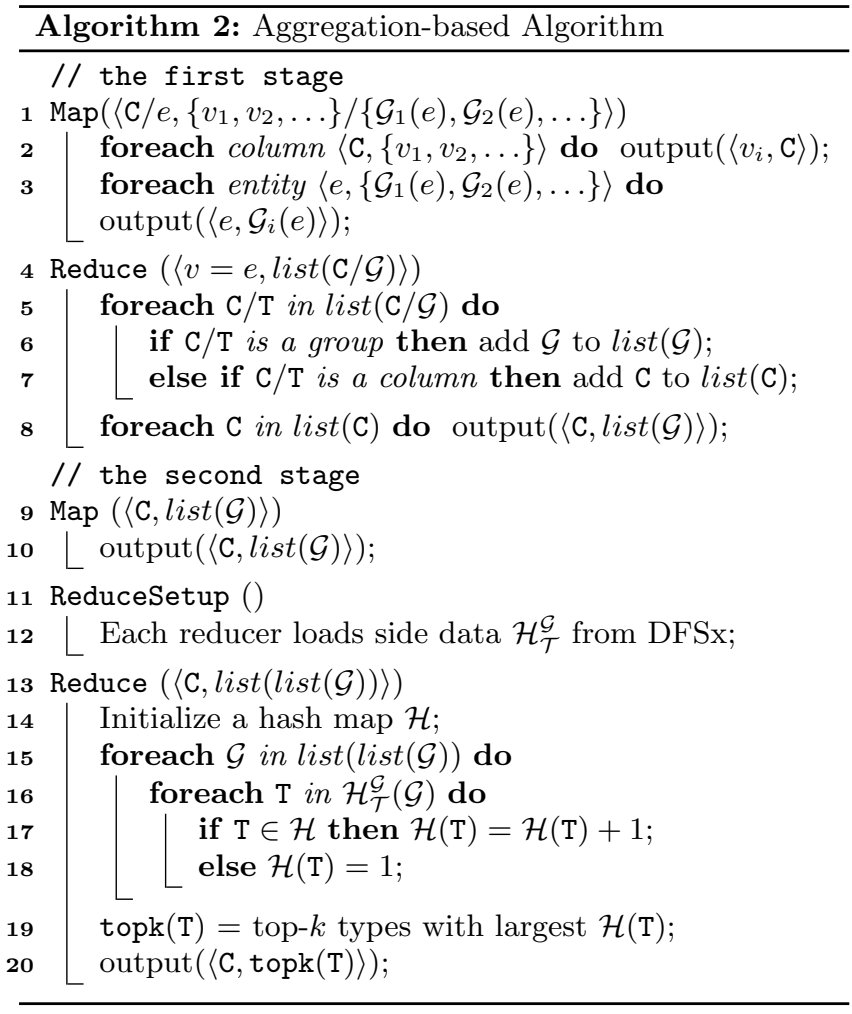

THEOREM 1. The aggregation based method satisfies correctness - it can correctly find top- $k$ types for each column.

Proof Sketch. The aggregation based method aggregates the types that contain an entity $e$ (i.e., $\mathcal{L}(e))$ into several disjoint groups $\mathcal{G}_{1}(e), \mathcal{G}_{2}(e), \ldots, \mathcal{G}_{l}(e)$. For each type $\mathrm{T}$ and column $\mathrm{C}$, the basic method counts their overlap based on $\langle e \in \mathrm{T}, \mathrm{T}\rangle$ and $\langle c \in \mathrm{C}, \mathrm{C}\rangle$. The aggregation method counts their overlap based on $\left\langle e, \mathcal{G}_{i}(e)\right\rangle$ and $\langle c, \mathrm{C}\rangle$. As $\cup_{1 \leq i \leq l} \mathcal{G}_{i}(e)=\mathcal{L}(e)$, and $\mathcal{G}_{i}(e) \cap \mathcal{G}_{j}(e)=\phi$ for $i \neq j$, the two counted overlap numbers are the same. Thus the aggregation based method satisfies correctness.

EXAMPLE 3. Consider three groups $\mathcal{G}_{1}=\left\{\mathrm{T}_{1}, \mathrm{~T}_{2}\right\}, \mathcal{G}_{2}=$ $\left\{\mathrm{T}_{1}, \mathrm{~T}_{3}\right\}, \mathcal{G}_{3}=\left\{\mathrm{T}_{1}, \mathrm{~T}_{4}\right\}$. Figure 4 shows how to use the aggregation based method to label each column. In the Map step of the first stage, for each entity, we generate <entity, group pairs. For $e_{1}$, we generate one pair $\left\langle e_{1}, \mathcal{G}_{1}\right\rangle$. The basic framework generates 16 key-value pairs for the knowledge base while the aggregation based method only generates 8 pairs. In the Reduce step, we find the groups of types that share common entities with columns. For $\mathrm{C}_{1}$, we get $\mathcal{G}_{1}, \mathcal{G}_{2}, \mathcal{G}_{3}$. In the Reduce step of the second stage, we utilize the side data $\mathcal{H}_{\mathcal{T}}^{\mathcal{G}}$ to compute overlap between each type and column. For example, consider $\left\langle\mathrm{C}_{1},\left\{\left\{\mathcal{G}_{1}\right\},\left\{\mathcal{G}_{1}\right\},\left\{\mathcal{G}_{2}\right\},\left\{\mathcal{G}_{2}\right\}\right\}\right\rangle$. Based on $\mathcal{G}_{1}=\left\{\mathrm{T}_{1}, \mathrm{~T}_{2}\right\}, \mathcal{G}_{2}=\left\{\mathrm{T}_{1}, \mathrm{~T}_{3}\right\}$, we have the overlap of $\mathrm{T}_{1}$ with $\mathrm{C}_{1}$ is 4 , and the overlap of $\mathrm{T}_{2}\left(\mathrm{~T}_{3}\right)$ with column $\mathrm{C}_{1}$ is 2 (2). Thus the top-1 type of column $\mathrm{C}_{1}$ is $\mathrm{T}_{1}$.

Discussion: The aggregation method can reduce the number of key-value pairs for knowledge bases at the expense of distributing the side data. Notice that distributing side data is more efficient than sending key-value pairs. This is because MapReduce has large overhead to generate the pairs, e.g., communication between job tracker and task tracker. 

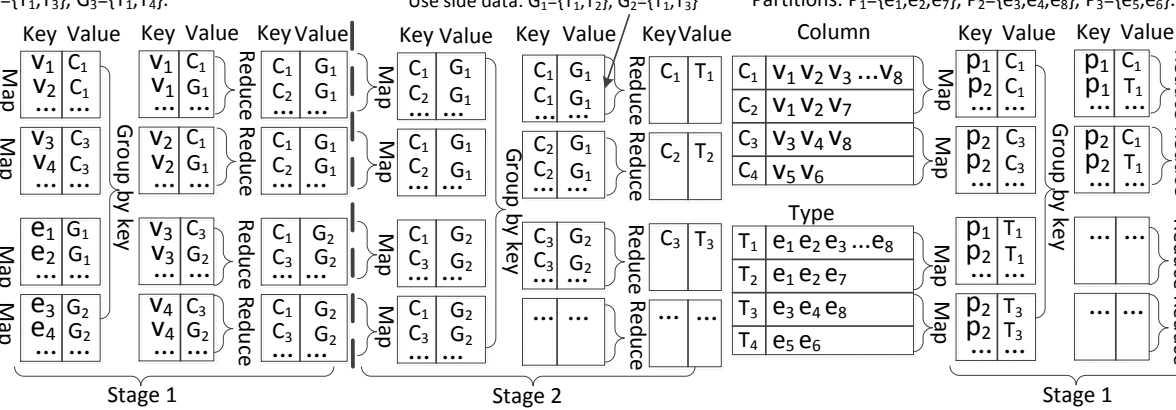

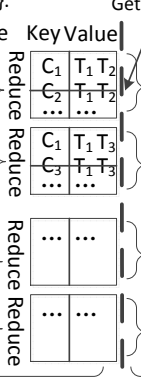

Stage 1

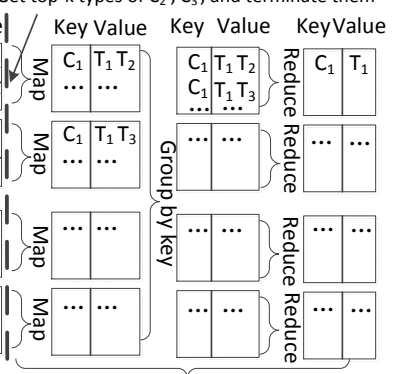

Figure 4: Knowledge type aggregation based method. Figure 5: Knowledge entity partition based method.

The aggregation method includes two parts: (1) using the groups to generate the key-value pairs for each entity; and (2) distributing the groups $\mathcal{H}_{\mathcal{T}}^{\mathcal{G}}$ to each node of the cluster. Our optimal objective is to select a good aggregation strategy to achieve the highest performance by trading-off them. Here we show two extreme aggregation strategies.

Case 1: For entity $e$, each type in $\mathcal{L}(e)$ is taken as a group. This strategy still produces large numbers of key-value pairs. However it does not need to distribute the side data.

Case 2: For entity $e$, the whole list $\mathcal{L}(e)$ is taken as a group. This method produces only one key-value pair for the entity. Thus it can minimize the number of key-value pairs. However it generates large numbers of distinct groups and the side data is very large.

The two strategies cannot balance the number of key-value pairs and the size of side data. To address this issue, we propose a cost model to quantify an aggregation strategy and utilize the cost model to select high-quality groups.

\subsection{Aggregation Cost Model}

The basic framework generates $\langle e, \mathrm{~T}\rangle$ key-value pairs. The aggregation based method reduces the number of key-value pairs by aggregating $\langle e, \mathrm{~T}\rangle$ pairs on types as follows. For each entity $e$, it generates a set of distinct groups, $\mathcal{G}_{1}(e), \mathcal{G}_{2}(e)$, $\ldots, \mathcal{G}_{l}(e)$. Let $\mathcal{G}_{1}, \mathcal{G}_{2}, \cdots, \mathcal{G}_{|\mathcal{G}|}$ denote the union of groups of all entities. Let $\mathrm{E}_{i}$ denote the set of entities whose groups include $\mathcal{G}_{i}$. That is $\mathrm{E}_{i}=\left\{e \mid \exists j, \mathcal{G}_{i}=\mathcal{G}_{j}(e)\right\}$. The aggregation based method distributes groups $\mathcal{G}_{1}, \mathcal{G}_{2}, \cdots, \mathcal{G}_{|\mathcal{G}|}$ as the side data. For each entity $e \in \mathrm{E}_{i}$, it generates key-value pairs $\left\langle e, \mathcal{G}_{i}\right\rangle$. Suppose the cost of processing a key-value pair in MapReduce is $\alpha$ and the cost of distributing a group $\mathcal{G}_{i}$ in the side data is $\beta\left|\mathcal{G}_{i}\right|$ where $\left|\mathcal{G}_{i}\right|$ is the number of types in $\mathcal{G}_{i}$. Thus the total cost of the aggregation based method is

$$
\sum_{i}\left(\alpha\left|\mathrm{E}_{i}\right|+\beta\left|\mathcal{G}_{i}\right|\right)
$$

and the total cost of the basic framework is

$$
\sum_{i}\left(\alpha\left|\mathrm{E}_{i}\right| \times\left|\mathcal{G}_{i}\right|\right)
$$

To select the optimal aggregation strategy for achieving the highest performance, we need to minimize Equation 1 . It is unlike to find the optimal aggregation strategy in polynomial time, as shown in Theorem 2

TheOREM 2. The problem of finding the optimal aggregation strategy is NP-hard.

Proof. We can prove the Theorem by a reduction from the 3-CNF-SAT problem. Due to space constraints, we omit the proof and interested readers refer to the full paper $¥$ for more details.

\footnotetext{
http://dbgroup.cs.tsinghua.edu.cn/ligl/ccd.pdf
}

Given Side-Data Budget: Usually each node has a memory budget $\mathcal{B}$ for the side data. Our goal is to fully utilize the budge $\mathcal{B}$ to accommodate aggregated groups so as to maximize the performance.

\subsection{Aggregation Algorithms}

Since the problem of finding the optimal aggregation strategy is NP-hard, we need to devise approximation algorithms. Consider any group $\mathcal{G}_{i}$. Based on Equations 1 and 2 the aggregation based method can reduce the cost by

$$
\alpha\left|\mathrm{E}_{i}\right| \times\left|\mathcal{G}_{i}\right|-\left(\alpha\left|\mathrm{E}_{i}\right|+\beta\left|\mathcal{G}_{i}\right|\right) .
$$

Based on this observation, we devise a greedy algorithm. For each entity $e$, we enumerate all possible groups on $\mathcal{L}(e)$. (Since the number of types that contain an entity is small, the number of possible groups is not large.) We union all possible groups for all entities and generate a possible group set. Then we enumerate each possible group $\mathcal{G}_{i}$ in the set and select the group with the largest benefit $\alpha\left|\mathrm{E}_{i}\right| \times\left|\mathcal{G}_{i}\right|-$ $\left(\alpha\left|E_{i}\right|+\beta\left|\mathcal{G}_{i}\right|\right)$. Then we remove the group and repeat the above step until the selected groups cover all entity-type pairs. If given a space budget $\mathcal{B}$, we terminate generating groups if the size of aggregated groups is larger than $\mathcal{B}$.

However there may be large numbers of possible groups, and it is expensive to enumerate all of them. To alleviate this problem, we utilize the knowledge structure to generate the groups as follows. We first pick a type T with no subtype. Let $\mathcal{G}_{i}$ denote the set of types that have a directed path to T (including itself). Obviously any type in $\mathcal{G}_{i}$ will take $\mathrm{T}$ as its subtype or has a descendant which takes $\mathrm{T}$ as a subtype. Let E denote the entity set of $\mathrm{T}$. We take $\mathcal{G}_{i}$ as a group. Then we remove the type $\mathrm{T}$ from the knowledge base and remove the entities in $\mathrm{E}$ from the entity sets of types in $\mathcal{G}_{i}$. We repeat the above steps until there is no type left (or the size of aggregated types is larger than the budget). Iteratively we can generate all groups. Notice that we need to build keyvalue pairs $\left\langle e,\left\{\mathcal{G}_{1}(e), \mathcal{G}_{2}(e), \ldots, \mathcal{G}_{l}(e)\right\}\right\rangle$ for the MapReduce input file and pairs $\left\langle\mathcal{G}_{i}, \mathcal{H}_{\mathcal{T}}^{\mathcal{G}}\left(\mathcal{G}_{i}\right)\right\rangle$ for the side data. We only need to slightly change our algorithm. For each pair $\left\langle\mathrm{E}, \mathcal{G}_{i}\right\rangle$, we generate pair $\left\langle\mathcal{G}_{i}, \mathcal{H}_{\mathcal{T}}^{\mathcal{G}}\left(\mathcal{G}_{i}\right)\right\rangle$. For each entity $e$ in $\mathrm{E}$, if there exists a pair with key $e$, we add $\mathcal{G}_{i}$ into its value; otherwise we generate a new pair $\left\langle e, \mathcal{G}_{i}\right\rangle$. Iteratively we can construct the two files (MapReduce input and side data).

For example, consider the four types in Figure 2 Types $\mathrm{T}_{2}, \mathrm{~T}_{3}, \mathrm{~T}_{4}$ are subtypes of type $\mathrm{T}_{1}$. Thus we can aggregate $\mathrm{T}_{2}$ with $\mathrm{T}_{1}$ and generate a group $\left\{\mathrm{T}_{1}, \mathrm{~T}_{2}\right\}$ which will be inserted into the side data $\mathcal{H}_{\mathcal{T}}^{\mathcal{G}}$. As $\mathrm{T}_{2}$ contains $\left\{e_{1}, e_{2}, e_{7}\right\}$, entities $e_{1}, e_{2}, e_{7}$ will use the group $\left\{\mathrm{T}_{1}, \mathrm{~T}_{2}\right\}$ and we will add the group into the MapReduce file.

Notice that our techniques do not dependent on type hierarchies, i.e., our methods still work if there is no type hierar- 
chy. On the other hand, we utilize type hierarchies heavily to improve the performance and scalability. We can easily extract hierarchies from YAGO and Freebase. For example, we can use the included type $\$$ associated with each Freebase type to construct a rich type hierarchy from Freebase. We can also identify the subtype relationships by comparing the entity sets of different types. (For example, if the entity set of a type contains that of another type, the latter type will be a subtype of the former one.)

\section{KNOWLEDGE ENTITY PARTITION}

If we can send similar 〈column, type〉 pairs into the same reducer and dissimilar $\langle$ column, type $\rangle$ pairs into different reducers, we can significantly improve the performance since we can prune large numbers of dissimilar 〈column, type〉 pairs. To this end, we propose a partition based method. We partition cell values of all columns into $p$ partitions $\mathrm{P}_{1}, \mathrm{P}_{2}, \ldots, \mathrm{P}_{p}$. If all the cell values in a column fall in the same partition, e.g., $\mathrm{P}_{i}$, we only use this partition to annotate the column and prune other unnecessary partitions. This method not only prunes many unnecessary partitions, but also reduces the number of key-value pairs since $\mathrm{P}_{i}$ may contain multiple cell values.

There are several research challenges. The first one is how to utilize the partitions to annotate each column. We will discuss a partition framework in Section 6.1. The second challenge is how to effectively partition the cell values. We study how to select a good partition strategy in Section 6.2. The third challenge is how to efficiently identify the corresponding partitions for each column and type, and we propose a bloom-filter based method in Section 6.3 .

\subsection{Partition Framework}

Let $\mathrm{V}$ denote the set of distinct cell values in all columns. We partition $\mathrm{V}$ into $p$ partitions $\mathrm{P}_{1}, \mathrm{P}_{2}, \ldots, \mathrm{P}_{p}$ such that (1) $\mathrm{P}_{i}$ is a subset of $\mathrm{V},(2) \cup_{1 \leq i \leq p} \mathrm{P}_{i}=\mathrm{V}$, and (3) $\mathrm{P}_{i} \cap \mathrm{P}_{j}=\phi$. We discuss partition algorithms in Section 6.2 Here we use partitions to label columns. We employ a two stage MapReduce framework and Algorithm 3 shows the pseudo-code. We take the basic framework as an example and our techniques can be easily integrated into the aggregation based method.

Stage 1: For each column, find the list of types that share at least one entity/cell value with the column.

Map: $\left\langle\mathrm{C},\left\{v_{1}, v_{2}, \ldots\right\}\right\rangle \rightarrow\left\langle\mathrm{P}_{i},\left[\mathrm{C}, \mathrm{P}_{i} \cap \mathrm{V}_{\mathrm{C}}, \mathrm{cnt}=\left|\mathrm{V}_{\mathrm{C}}\right|-\mid \mathrm{P}_{i} \cap \mathrm{V}_{\mathrm{C}}\right]\right\rangle$. $\left\langle\mathrm{T},\left\{e_{1}, e_{2}, \ldots\right\}\right\rangle \rightarrow\left\langle\mathrm{P}_{i},\left[\mathrm{~T}, \mathrm{P}_{i} \cap \mathrm{E}_{\mathrm{T}}\right]\right\rangle$.

For each column $\mathrm{C}$, it identifies the partitions that contain cell values in the column. Since there are large numbers of cell values that cannot be loaded into memory, it is not straightforward to identify the partitions and we will discuss efficient techniques in Section 6.3. Suppose we get $k$ partitions $\mathrm{P}_{1}, \mathrm{P}_{2}, \ldots, \mathrm{P}_{k}$. Notice that if a cell value in $\mathrm{P}_{i}$ is not an entity in the knowledge base, we do not need to distribute the cell value. In other words, we only distribute the cell values in $\mathrm{C}$ that also appear in knowledge types. For simplicity, we use $\mathrm{V}_{\mathrm{C}}^{\mathrm{T}}$ to denote the set of such cell values. Thus we generate the following key-value pairs

$$
\left\langle\mathrm{P}_{i},\left[\mathrm{C}, \mathrm{P}_{i} \cap \mathrm{V}_{\mathrm{C}}^{\mathrm{T}}, \mathrm{cnt}=\left|\mathrm{V}_{\mathrm{C}}^{\mathrm{T}}\right|-\mid \mathrm{P}_{i} \cap \mathrm{V}_{\mathrm{C}}^{\mathrm{T}}\right]\right\rangle
$$

for $1 \leq i \leq k$, where $\mathrm{cnt}$ is the number of entities that are in the column but not in the partition. Obviously if $\mathrm{cnt}=0$, all the cell values appear in partition $\mathrm{P}_{i}$. In the Reduce

\footnotetext{
http://wiki.freebase.com/wiki/Included_Type
}

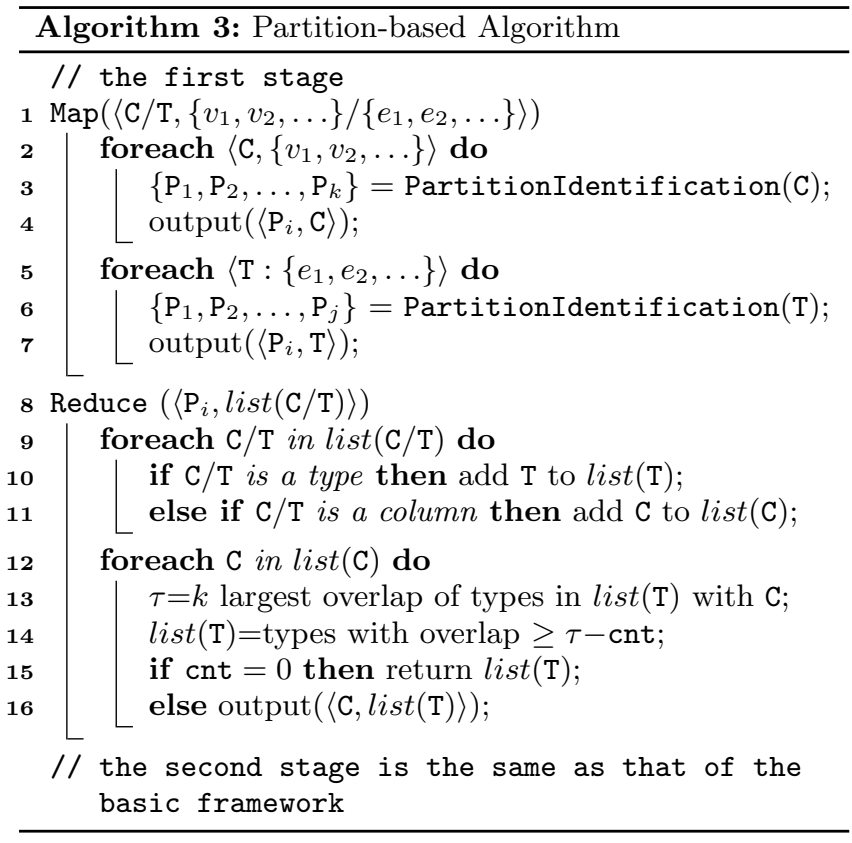

step, we can use this partition to directly label the column, because all of types that share entities with the column must fall in this partition.

Similarly, for each type T, it identifies the partitions that contain entities in the type. Suppose we get $j$ partitions $\mathrm{P}_{1}, \mathrm{P}_{2}, \ldots, \mathrm{P}_{j}$. We generate the following key-value pairs

$$
\left\langle\mathrm{P}_{i},\left[\mathrm{~T}, \mathrm{P}_{i} \cap \mathrm{E}_{\mathrm{T}}\right]\right\rangle
$$

for $1 \leq i \leq j$, where $\mathrm{E}_{\mathrm{T}}$ is the entity set of type $\mathrm{T}$.

Reduce: $\left\langle\mathrm{P}_{i}\right.$, list $\left.(\mathrm{C} / \mathrm{T})\right\rangle \rightarrow\langle\mathrm{C}, \operatorname{list}(\mathrm{T})\rangle$.

For each partition, we get a list of columns and types. We first extract the list of columns and the list of types. Then for each column $\mathrm{C}$, we scan the type list and for each type $\mathrm{T}$, we compute $\left(\mathrm{P}_{i} \cap \mathrm{V}_{\mathrm{C}}^{\mathrm{T}}\right) \cap\left(\mathrm{P}_{i} \cap \mathrm{E}_{\mathrm{T}}\right)$, which is the overlap similarity of $\mathrm{C}$ and $\mathrm{T}$ in the partition. Let $\tau$ denote the $k$-th largest overlap similarly among all types with column C. Since column C contains at most other cnt entities besides the partition, each type $\mathrm{T}$ at most includes other cnt cell values of $\mathrm{C}$. Thus we can prune the types with the overlap smaller than $\tau-$ cnt. In other words, we generate a list of types, $\operatorname{list}(\mathrm{T})$, where each type has no smaller than $\tau-$ cnt overlap with the column, and output key-value pairs $\langle\mathrm{C}, \operatorname{list}(\mathrm{T})\rangle$. Especially, if $\mathrm{cnt}=0$, column $\mathrm{C}$ contains no other cell value and all types having overlap with $\mathrm{C}$ are also in this partition, thus we can directly use the partition in this reducer to label the column and do not need to output the pair.

Stage 2: Compute top- $k$ types of every column. It is the same as that of the basic framework.

EXAmPle 4. Suppose we partition the entities into three partitions $\mathrm{P}_{1}=\left\{e_{1}, e_{2}, e_{7}\right\}, \mathrm{P}_{2}=\left\{e_{3}, e_{4}, e_{8}\right\}, \mathrm{P}_{3}=\left\{e_{5}, e_{6}\right\}$. Figure 5 illustrates a running example of using the partitionbased method to label the columns. In the Map step of the first stage, it generates $\langle$ partition, entity $\rangle$ and $\langle$ partition, column $\rangle$ pairs. For $\mathrm{T}_{1}$, it generates three pairs $\left\langle\mathrm{P}_{1}, \mathrm{~T}_{1}\right\rangle,\left\langle\mathrm{P}_{2}, \mathrm{~T}_{1}\right\rangle,\left\langle\mathrm{P}_{3}, \mathrm{~T}_{1}\right\rangle$. The basic framework generates 16 pairs for the knowledge base while the partition based method only generates 6 pairs. For column $\mathrm{C}_{2}$, it generates one pair $\left\langle\mathrm{P}_{1}, \mathrm{C}_{2}\right\rangle$. As all cell 
values fall in partition $\mathrm{P}_{1}$, we can use this partition to label the column in the Reduce step. Similarly all cell values in $\mathrm{C}_{3}$ fall in partition $\mathrm{P}_{2}$. The basic framework generates 16 pairs for the columns while the partition method only generates 4 pairs $\left(v_{5}, v_{6}, v_{7}, v_{8}\right.$ do not appear in the knowledge base). In the Reduce step, in the reducer of $\mathrm{P}_{1}$, we can directly label $\mathrm{C}_{2}$ and do not need to output the key-value pairs since all types having overlap with $\mathrm{C}_{2}$ are also in this partition. Similarly we can directly label $\mathrm{C}_{3}$ and early terminate the two columns.

The partition based method is orthogonal to the aggregation based method and two methods can be used simultaneously.

\subsection{Partition Algorithm}

Since different partition strategies will affect the pruning power (whether we can prune unnecessary partitions) and the number of key-value pairs, it is very important to select a high-quality partition strategy. To address this issue, we first study how to evaluate a partition strategy.

To evaluate a partition strategy, we model the columns as a graph and transform the graph partition problem to our partition problem as follows. For ease of presentation, we first assume each column has at most two cell values. In the graph, the vertices are cell values. There is an edge between two vertices if there is a column which contains the cell values of the two vertices. The edge weight is the number of columns that contain the two cell values. If we want to partition the cell values into $p$ partitions, we aim to partition the graph into $p$ subgraphs. Notice that in a graph partition strategy, if we cut an edge with weight $w$, $w$ columns w.r.t. the edge will be affected. Thus cutting an edge with weight $w$ will increase $w$ key-value pairs. It also reduces the probability to use a single partition to label a column. Thus we want to find a partition with minimum cut weight, i.e., the sum of the weights of cut edges. Therefore, our finding optimal partition strategy problem is NP-Hard proved by a reduction from the graph partition problem.

We extend this method to support the case that each column can have more than two cell values. The only difference is that the weight of cutting an edge is dependent on those edges that have been cut. We also utilize the graph partition method to evaluate a partition strategy. However Web tables are not given and we cannot partition the cell values off-line. To address this issue, we use entities in the knowledge base to facilitate the partition. The main reason is that if a cell value does not appear in the knowledge base, we cannot utilize it to do annotation. Thus we can utilize entities to construct the graph. In addition, given an edge of two entities, we cannot get its weight, i.e., the real number of columns that contain the two entities. Alternatively, we use the number of types that contain the two entities to approximate the edge weight. Thus we can build a graph based on the knowledge base. We extend existing graph-partitioning algorithms (e.g., 16]) to partition the weighted graph, with the goal of (1) minimizing the weight of cut edges and (2) making each partition have the same weight.

Figure 6 shows the graph constructed from the knowledge base in Figure 2. Each vertex represents a distinct entity. The weight of an edge is the number of types that contain the two entities on the edge. The weight of edges between two dotted ellipses denotes the weights of all the edges between vertices in the two ellipses. If $p=3$, the three partitions should be $\mathrm{P}_{1}=\left\{e_{1}, e_{2}, e_{7}\right\}, \mathrm{P}_{2}=\left\{e_{3}, e_{4}, e_{8}\right\}, \mathrm{P}_{3}=\left\{e_{5}, e_{6}\right\}$.

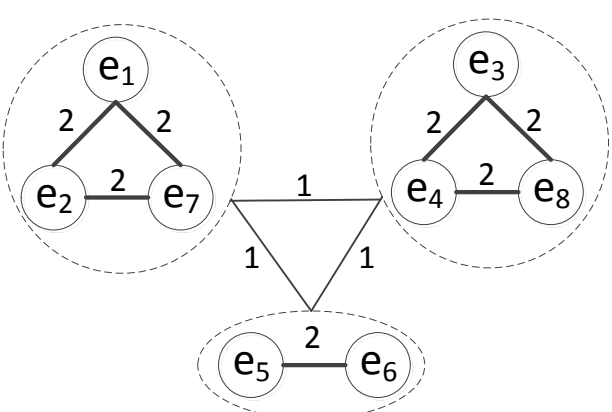

Figure 6: Partitioning entities.

\subsection{Partition Identification}

In this section, we discuss how to identify partitions for each type and column. For each type, during the graph partitioning phase, we keep the partitions for each type and generate the key-value pair $\left\langle\mathrm{T},\left\{\mathrm{P}_{1}, \mathrm{P}_{2}, \ldots\right\}\right\rangle$. Thus in the Map step of the first stage, we directly load the pairs to generate the key-value pairs $\left\langle\mathrm{P}_{i}, \mathrm{~T}\right\rangle$.

Since each column is not given, we need to online identify the partitions that contain a cell value in the column. A straightforward method is to use a hash based method. However the number of entities is rather large, and we cannot load the hash table into memory. To address this issue, we use a bloom filter based method. The bloom filter [4] is a probabilistic method to efficiently check whether an element is in a set using multiple hash functions.

For each partition, we use a bloom filter to represent the entities in the partition. To identify the partitions of each column, for each cell value, we enumerate the $p$ partitions. For each partition, we check whether the cell value is in the partition. If the cell value is not in the partition based on the bloom filter, we can ignore the partition; otherwise the partition is added into the partition list of the column, with a false-positive probability. Iteratively we can identify all the partitions. The false positives introduced by the bloom filter will not affect the correctness as stated in Theorem 3

THEOREM 3. The partition based method satisfies correctness - it can correctly find top-k types for each column.

Proof Sketch. The partition based method partitions the types into $\mathrm{P}_{1}, \mathrm{P}_{2}, \ldots, \mathrm{P}_{p}$. If a cell value is the same as an entity, they must be in a same partition. Thus if a type $\mathrm{T}$ and a column $\mathrm{C}$ share a common entity/cell value, they must share a common partition. Although the bloom filter may involve false positives, we compute their overlap based on $\left(\mathrm{P}_{i} \cap \mathrm{V}_{\mathrm{C}}^{\mathrm{T}}\right) \cap\left(\mathrm{P}_{i} \cap \mathrm{E}_{\mathrm{T}}\right)$. If a cell value is a false positive of partition $\mathrm{P}_{i}$, it will not be in $\mathrm{P}_{i} \cap \mathrm{E}_{\mathrm{T}}$. Thus the partition based method satisfies correctness.

However this method needs to enumerate $p$ bloom filters of every partition and for each partition, it has to compute $t$ hash values using the $t$ hash functions, which is expensive. To alleviate this problem, we utilize a bloom filter hierarchy 25] to improve the performance. We build a binary tree structure where each leaf node is a bloom filter. The parent of two leaf nodes is the bloom filter build upon entities in the two partitions. Obviously the root is the bloom filter for all entities. Then given a cell value, we identify the partitions in an up-down manner from the root. If the cell value is not in the root bloom filter, we terminate; otherwise we check its two children. Iteratively, we can identify all partitions. This method only needs to enumerate $\log _{2} p$ bloom filters. 


\section{EXPERIMENTS}

The goals of our experiments are to (1) evaluate the effectiveness of our column concept identification approach that utilizes knowledge bases fuzzily to label Web tables; and (2) validate the performance and scalability of our methods.

Datasets: Table 2 summarizes our datasets. For Web tables, we used (1) WWT whose tables were manually extracted from Wikipedia and annotated with ground-true entities and types, and (2) WEX from Wikipedia by a Freebase Wikipedia Extraction project. For knowledge bases, we used (1) Freebase ** $^{* *}$ a collaboratively created knowledge based that is manually built by data-loving users, and (2) Yagd ${ }^{\dagger \dagger}$ a large semantic knowledge base which is derived from Wikipedia, WordNet and GeoNames. Both knowledge bases are publicly well-known.

Table 2: Web Tables and Knowledge Bases

\begin{tabular}{|r|r|r|r|r|}
\hline Dataset & Size (MB) & \# Tables & \# Columns & \# Cell Values \\
\hline WWT & 7.3 & 6318 & 27,695 & 459,641 \\
\hline WEX & 423 & 242,062 & $1,179,125$ & $19,922,138$ \\
\hline \hline Dataset & Size (MB) & \# Types & \# Entities & \\
\cline { 2 - 4 } Freebase & 1200 & 1721 & $43,648,334$ & \\
\cline { 1 - 3 } Yago & 157 & 292,861 & $9,003,751$ & \\
\cline { 1 - 2 } & & &
\end{tabular}

Settings: We implemented our algorithms in Hadoop. All experiments except scaleup ones were run on a 20-node Dell cluster, each with two Intel(R) Xeon(R) E5420 2.5GHZ processors with eight cores, 16GB RAM, and 1TB disk. Thus the cluster consists of 160 cores, 320GB memory and 20TB disk. Each node is installed with 64-bit Ubuntu Server 10.04, Java 1.6, and Hadoop 1.0.3. We set $\mathcal{B}$ as 1GB. $\alpha$ and $\beta$ can be set by evaluating the cost of distributing key-value pairs and side data based on experimental evaluation. In our experiments, we set $\beta$ as 1 and $\alpha$ as the ratio of the number of entities to the number of distinct entities.

Due to space constraints, in the experiments we only showed the results on the overlap similarity, Jaccard similarity, Fuzzy Jaccard similarity. We obtained similar results for other similarity functions, e.g., Cosine and Dice.

\subsection{Annotation Quality}

In this section, we evaluate the annotation quality of our similarity-join based framework. We compared with the machine learning based method [19], GraphicalModel. We used the well-known metrics, precision, recall, and F-measure, to evaluate the annotation quality. Let $c_{n o}$ denote the number of columns that have been manually annotated with ground truth, $a_{n o}$ denote the number of annotated columns of an algorithm, and $a_{t}$ denote the number of truly annotated columns (If the ground truth of a column is in the top- $k$ types annotated by an algorithm, the column is taken as truly annotated.). The precision is $p=\frac{a_{t}}{a_{n o}}$, the recall is $r=\frac{a_{\mathrm{t}}}{c_{\mathrm{no}}}$, and the F-measure is $\frac{2 \cdot p \cdot r}{p+r}$.

We first compared the quantity by varying $k$ on the WWT and Freebase datasets. Figure 7 shows the result quality. We have the following observations. First, our similarity join based method, especially using Jaccard similarity and fuzzy Jaccard similarity, nearly achieved the same quality as

\footnotetext{
http://www.it.iitb.ac.in/ sunita/wwt/

http://wiki.freebase.com/wiki/WEX

** http://www.freebase.com

th http://www.mpi-inf.mpg.de/yago-naga/yago/
}

the machine learning based method GraphicalModel. Second, for precision, our method using fuzzy Jaccard similarity outperformed GraphicalModel. This is because for each column, GraphicalModel will identify top- $k$ types which may be wrongly annotated while our method will not annotate the columns if it cannot find relevant types. In terms of recall, GraphicalModel outperformed our similarity join based method since it can always identify top- $k$ types for each column. Third, our method using fuzzy Jaccard similarity was better than that using Jaccard similarity, which in turn outperformed that using the overlap similarity. The is because fuzzy Jaccard similarity can tolerate inconsistences between cell values and entities, and Jaccard similarity utilized the type and column sizes to annotate a column which can remove the stop types, e.g., music. For example, fuzzy Jaccard similarity outperformed Jaccard similarity about 5\%, which was better than overlap similarity about $5 \%$. Fourth, a larger $k$ will increase the annotation quality, because it returned more types to annotate a column.

We then evaluated the quality on different datasets by fixing $k=3$. Figure 8 shows the results. We have the following observations. First, on different Web tables and knowledge bases, our similarity join based method still achieved the same high quality as GraphicalModel. Second, the annotation quality using Yago is better than that using Freebase, because Yago contains larger numbers of types than Freebase.

\subsection{Performance and Scalability}

To evaluate the performance of our algorithms on large datasets, we increased WEX by $5,10,15,20,25$ times as follows. Suppose we want to increase a dataset by $n$ times. For each column with $m$ cell values, we randomly selected $m-1, m-2, \ldots, m-n$ cell values and took them as new columns. (If a column had smaller than $n$ cell values, we randomly selected $n$ groups of $m-1$ cell values and took them as new columns.) We use WEX $\times n$ to represent the increase factor. For example, WEX $\times 10$ represents the WEX dataset increased 10 times. Notice that WEX $\times 25$ contains about 30 million columns and 500 million cell values.

\subsubsection{Running Time}

We compare the annotation performance of our four methods: the basic similarity-join framework (Basic), the knowledge type aggregation based method (Aggregation), the knowledge entity partition based method (Partition), and the hybrid method which uses these two optimization techniques (Hybrid). Notice that GraphicalModel is rather inefficient. For example, it took an hour to annotate 100 tables using Freebase. Thus we did not compare with it in terms of running time. We compared the performance by varying the dataset sizes. Since overlap similarity and Jaccard similarity nearly achieved the same performance, we only used the Jaccard and fuzzy Jaccard similarity. We used WEX and Freebase datasets and set $k=3$. Figure 9 shows the results. In this figure, for each algorithm, the bottom bar denotes the time of the first stage in the algorithm and the top bar denotes the time of the second stage.

We can see that the Hybrid method achieved the highest performance and the Basic method got the worst performance. The knowledge type aggregation based method improved the performance as it can reduce the number of keyvalues pairs by aggregating the types. The knowledge entity partition based method also improved the performance, because it cannot only prune large numbers of unnecessary 


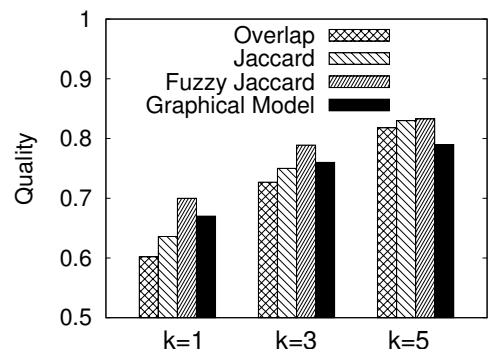

(a) Precision.

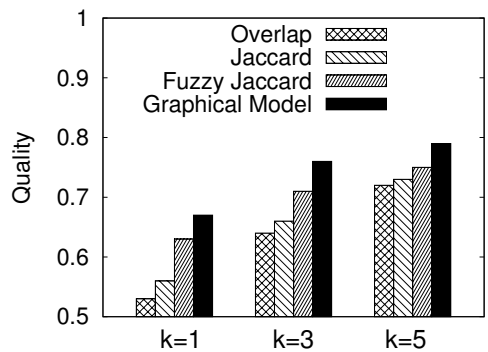

(b) Recall.

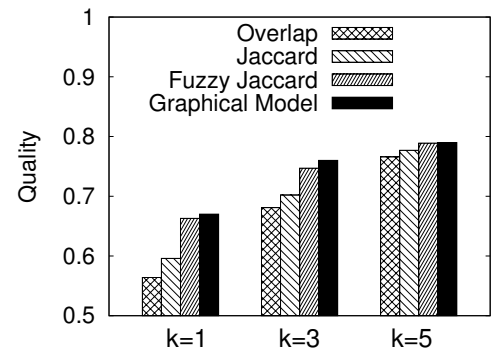

(c) F-measure.

Figure 7: Evaluating annotation quality by varying $k$ on WWT and Freebase datasets.

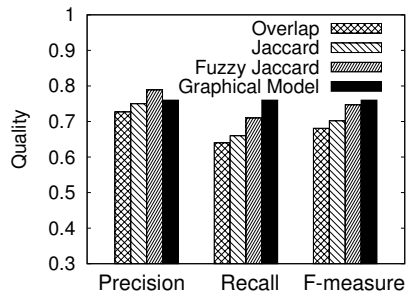

(a) WWT + Freebase

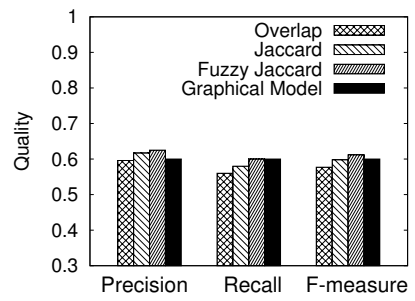

(b) WEX + Freebase

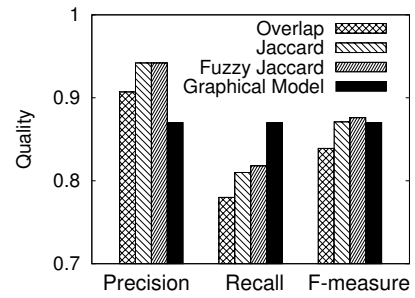

(c) WWT + Yago

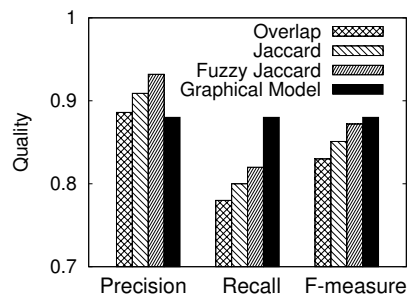

(d) WEX + Yago

Figure 8: Evaluating annotation quality on different datasets $(k=3)$.

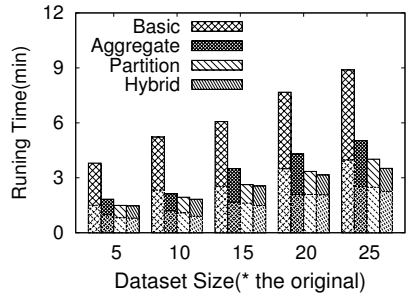

(a) Jaccard

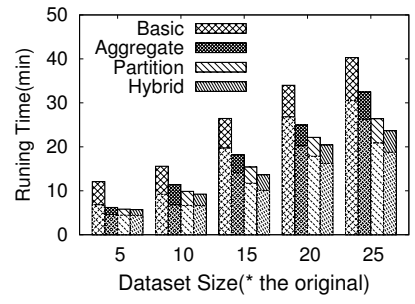

(b) Fuzzy Jaccard

Figure 9: Evaluating running time by varying dataset sizes on WEX + Freebase $(k=3)$.

partitions but also reduce the number of key-value pairs. For example, on WEX $\times 25$ dataset, Basic took about 9 minutes, Aggregation improved the time to 5 minutes, and Partition took about 4 minutes. Hybrid further improved the time to 3.5 minutes. The experimental results show the superiority of our proposed techniques which can significantly improve the performance.

\subsubsection{Speedup}

We evaluated our algorithms by fixing the datasset size and varying the number of nodes in the cluster. We used WEX $\times 10$ and Freebase datasets. Figure 10 shows the running time by varying the number of nodes from 4 to 20 . We can see that with the increase of the number of nodes, the performance of our method improved. For instance, using Jaccard similarity, Hybrid took 4.5 minutes on 4 nodes and improved the time to less than 2 minutes for 20 nodes.

We also evaluated the speedup of our algorithms, i.e., the ratio of the performance improvement compared with that of the minimum number of nodes. For example, for 20 nodes, the speedup is the ratio between the running time on the 4 nodes and that on 20 nodes. We also plot the ideal speedup curve. For example, if the nodes are doubled, the ideal speedup should be 2 . Figure 11 shows the results. We can see that our methods achieved good speedup.

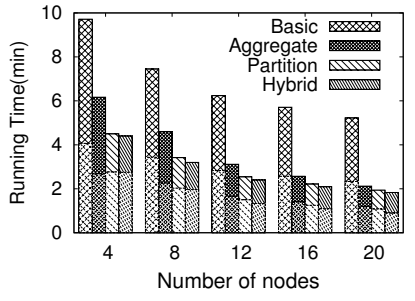

(a) Jaccard (b) Fuzzy Jaccard

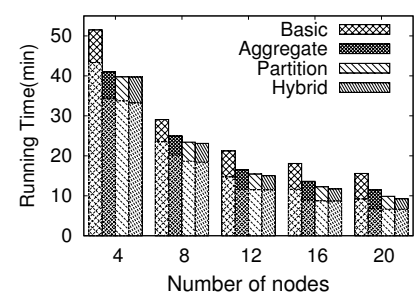

Figure 10: Evaluating running time by varying number of nodes on WEX $\times \mathbf{1 0}+$ Freebase $(k=3)$.

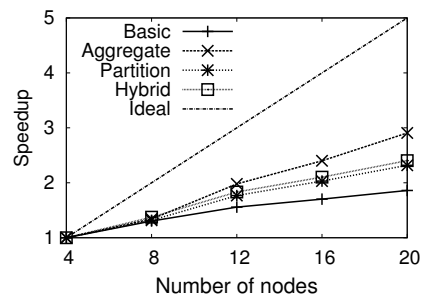

(a) Jaccard

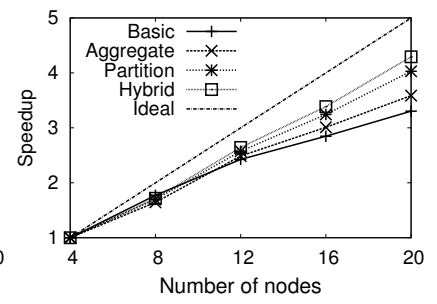

(b) Fuzzy Jaccard
Figure 11: Speedup on WEX $\times 10+$ Freebase $(k=3)$.

\subsubsection{Scaleup}

To evaluate the scaleup of our methods, we increased both the dataset sizes and the number of nodes in the cluster. We used our best algorithm, Hybrid, which used both knowledge type aggregation and entity partition techniques. We also added the ideal scaleup curve which should be a constant. Figures 12 and 13 show the running time on the Freebase and Yago datasets respectively, with increasing the number of nodes from 4 to 20 and dataset sizes from 5 to 25 . We can see that our method scaled up very well for both Jaccard similarity and fuzzy Jaccard similarity, and nearly reached the ideal scaleup. This is attributed to our partition based method which can prune unnecessary partitions and reduce the number of key-value pairs. 


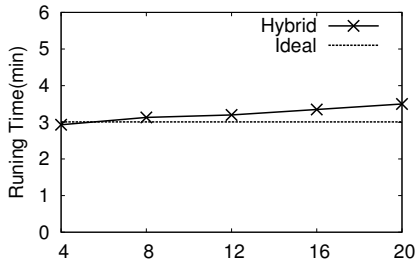

Number of nodes and dataset size $(1.25 \mathrm{x})$

(a) Jaccard

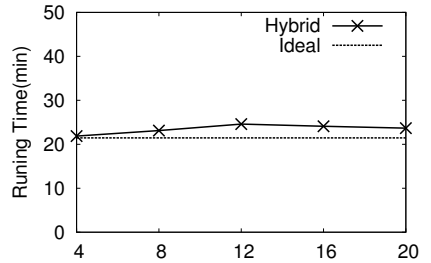

Number of nodes and dataset size(1.25x)

(b) Fuzzy Jaccard
Figure 12: Scaleup by increasing the dataset sizes and the number of nodes on WEX + Freebase $(k=3)$.

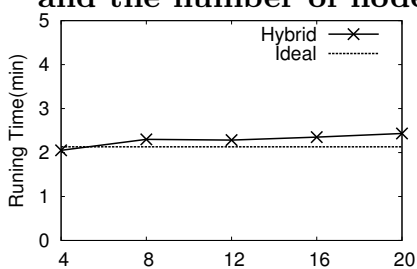

Number of nodes and dataset size $(1.25 \mathrm{x})$

(a) Jaccard

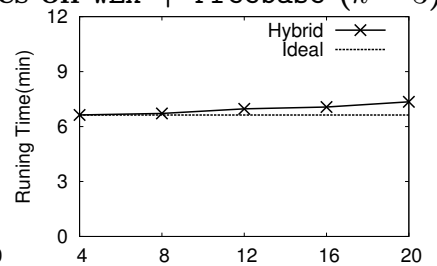

Number of nodes and dataset size(1.25x)

(b) Fuzzy Jaccard
Figure 13: Scaleup by increasing the dataset sizes and the number of nodes on WEX + Yago $(k=3)$.

\section{CONCLUSION}

We studied the column concept determination problem for Web tables using knowledge bases. We enabled fuzzy matching between the cell content and knowledge entities. We proposed a knowledge type aggregation based method which aggregated the types into groups and shared the groups for different entities to reduce the number of key-value pairs. We proved that the problem of finding the optimal aggregation strategy is NP-hard. We utilized the knowledge hierarchy to effectively aggregate types. We proposed a knowledge entity partition based method which partitioned the entities into different partitions. For each column, we identified its partitions and used the types falling in these partitions to annotate the column. We proposed a graph partition based algorithm to generate high-quality partitions. Experimental results on real datasets show that our algorithm achieved high annotation quality and performance and scaled well.

\section{ACKNOWLEDGMENTS}

Guoliang $\mathrm{Li}$ is partly supported by the National Natural Science Foundation of China under Grant No. 61003004, 61272090, National Grand Fundamental Research 973 Program of China under Grant No. 2011CB302206, a project of Tsinghua University under Grant No. 20111081073, Tsinghua-Tencent Joint Laboratory for Internet Innovation Technology, and the "NExT Research Center" funded by MDA, Singapore, under Grant No. WBS:R-252-300-001-490. Jian Li is supported in part by the National Basic Research Program of China Grant 2011CBA00300, 2011CBA00301, the National Natural Science Foundation of China Grant 61202009, 61033001, 61061130540, 61073174.

\section{REFERENCES}

1] S. Abiteboul, E. Antoine, and J. Stoyanovich. Viewing the web as a distributed knowledge base. In ICDE, 2012.

[2] A. Arasu, V. Ganti, and R. Kaushik. Efficient exact set-similarity joins. In $V L D B, 2006$.

[3] S. Auer, C. Bizer, G. Kobilarov, J. Lehmann, R. Cyganiak, and Z. G. Ives. Dbpedia: A nucleus for a web of open data. In $I S W C / A S W C$, pages 722-735, 2007.

[4] B. H. Bloom. Space/time trade-offs in hash coding with allowable errors. Commun. ACM, 13(7):422-426, 1970.

[5] K. D. Bollacker, C. Evans, P. Paritosh, T. Sturge, and J. Taylor. Freebase: a collaboratively created graph database for structuring human knowledge. In SIGMOD, 2008.

[6] M. J. Cafarella, A. Y. Halevy, D. Z. Wang, E. Wu, and Y. Zhang. Webtables: exploring the power of tables on the web. PVLDB, 1(1):538-549, 2008.
[7] S. Chaudhuri, others Robust and efficient fuzzy match for online data cleaning. In SIGMOD, 2003.

[8] J. Dean and S. Ghemawat. Mapreduce: Simplified data processing on large clusters. In OSDI, 2004.

[9] H. Elmeleegy, J. Madhavan, and A. Y. Halevy. Harvesting relational tables from lists on the web. $P V L D B$, 2(1):1078-1089, 2009.

[10] Google. Introducing knowledge graph. http://insidesearch.blogspot.com/2012/05/introducingknowledge-graph-things-not.html.

[11] L. Gravano, P. G. Ipeirotis, H. V. Jagadish, N. Koudas, S. Muthukrishnan, and D. Srivastava. Approximate string joins in a database (almost) for free. In $V L D B$, pages 491-500, 2001.

[12] X. Guo, Y. Chen, J. Chen, and X. Du. ITEM: Extract and integrate entities from tabular data to RDF knowledge base. In $A P W e b, 2011$.

[13] R. Gupta and S. Sarawagi. Answering table augmentation queries from unstructured lists on the web. $P V L D B$, 2(1):289-300, 2009.

[14] L. Han, T. Finin, C. S. Parr, J. Sachs, and A. Joshi. Rdf123: From spreadsheets to rdf. In $I S W C, 2008$.

[15] G. Hignette, P. Buche, J. Dibie-Barthélemy, and O. Haemmerlé. Fuzzy annotation of web data tables driven by a domain ontology. In $E S W C, 2009$.

[16] G. Karypis and V. Kumar. Multilevel k-way hypergraph partitioning. In $D A C, 1999$.

[17] Y. Kim and K. Shim. Parallel top-k similarity join algorithms using mapreduce. In ICDE, 2012.

[18] G. Li, D. Deng, J. Wang, and J. Feng. Pass-join: A partition-based method for similarity joins. $V L D B$, pages 253-264, 2011.

[19] G. Limaye, S. Sarawagi, and S. Chakrabarti. Annotating and searching web tables using entities, types and relationships. PVLDB, 3(1):1338-1347, 2010.

[20] X. Ling, A. Halevy, F. Wu, and C. Yu. Synthesizing union tables from the web. In IJCAI, 2013.

[21] A. Metwally and C. Faloutsos. V-smart-join: A scalable mapreduce framework for all-pair similarity joins of multisets and vectors. PVLDB, 5(8):704-715, 2012.

[22] R. Pimplikar and S. Sarawagi. Answering table queries on the web using column keywords. PVLDB, 5(10):908-919, 2012.

[23] G. Quercini and C. Reynaud. Entity discovery and annotation in tables. In $E D B T, 2013$.

[24] S. Sarawagi and A. Kirpal. Efficient set joins on similarity predicates. In SIGMOD, 2004.

[25] K. Shanmugasund., H. Brönnimann, and N. Memon. Payload attribution via hierarchical bloom filters. In CCS, 2004.

[26] F. M. Suchanek, G. Kasneci, and G. Weikum. Yago: a core of semantic knowledge. In $W W W, 2007$.

[27] M. van Assem, others Converting and annotating quantitative data tables. In $I S W C, 2010$.

[28] P. Venetis, A. Y. Halevy, J. Madhavan, M. Pasca, W. Shen, F. Wu, G. Miao, and C. Wu. Recovering semantics of tables on the web. PVLDB, 4(9):528-538, 2011.

[29] R. Vernica, M. J. Carey, and C. Li. Efficient parallel set-similarity joins using mapreduce. In SIGMOD, 2010.

[30] J. Wang, G. Li, and J. Feng. Trie-join: Efficient trie-based string similarity joins with edit-distance constraints. $P V L D B$, 3(1):1219-1230, 2010.

[31] J. Wang, G. Li, and J. Feng. Fast-join: An efficient method for fuzzy token matching based string similarity join. In ICDE, 2011.

[32] J. Wang, G. Li, and J. Feng. Can we beat the prefix filtering?: an adaptive framework for similarity join and search. In SIGMOD Conference, pages 85-96, 2012.

[33] J. Wang, H. Wang, Z. Wang, and K. Q. Zhu. Understanding tables on the web. In ER, 2012.

[34] $\mathrm{W}$. Wu, others Probase: a probabilistic taxonomy for text understanding. In SIGMOD, 2012.

[35] C. Xiao, W. Wang, and X. Lin. Ed-join: an efficient algorithm for similarity joins with edit distance constraints. PVLDB, 1(1):933-944, 2008.

[36] C. Xiao, W. Wang, X. Lin, and J. X. Yu. Efficient similarity joins for near duplicate detection. In $W W W, 2008$.

[37] M. Yakout, K. Ganjam, K. Chakrabarti, and S. Chaudhuri. Infogather: entity augmentation and attribute discovery by holistic matching with web tables. In SIGMOD, 2012.

[38] C. Yu. Towards a high quality and web-scalable table search engine. In $K E Y S, 2012$. 